\title{
A Cold-Atom Interferometer with Microfabricated Gratings and a Single Seed Laser
}

Jongmin Lee ( $\nabla$ neoscien@gmail.com )

Sandia National Laboratories https://orcid.org/0000-0002-7084-5530

Roger Ding

Sandia National Laboratories

Justin Christensen

Sandia National Laboratories

Randy Rosenthal

Sandia National Laboratories

Aaron Ison

Sandia National Laboratories

Daniel Gillund

Sandia National Laboratories

David Bossert

Sandia National Laboratories

Kyle Fuerschbach

Sandia National Laboratories

William Kindel

Sandia National Laboratories

Patrick Finnegan

Sandia National Laboratories

Joel Wendt

Sandia National Laboratory

Michael Gehl

Sandia National Laboratories

Hayden McGuinness

Sandia National Laboratories

Charles Walker

Sandia National Laboratories

Anthony Lentine

Sandia National Laboratory

Shanalyn Kemme

Sandia National Laboratories

Grant Biedermann 
University of Oklahoma

Peter Schwindt

Sandia National Laboratories

\section{Article}

Keywords: miniaturization, GMOT, cold-atom interferometer accelerometer

Posted Date: August 9th, 2021

DOl: https://doi.org/10.21203/rs.3.rs-736276/v1

License: (c) (i) This work is licensed under a Creative Commons Attribution 4.0 International License. Read Full License

Version of Record: A version of this preprint was published at Nature Communications on September 1st, 2022. See the published version at https://doi.org/10.1038/s41467-022-31410-4. 


\title{
A Cold-Atom Interferometer with Microfabricated Gratings and a Single Seed Laser
}

\author{
Jongmin Lee ${ }^{1, *}$, Roger Ding ${ }^{1}$, Justin Christensen ${ }^{1}$, Randy R. Rosenthal ${ }^{1}$, Aaron Ison ${ }^{1}$, \\ Daniel P. Gillund ${ }^{1}$, David Bossert ${ }^{1}$, Kyle H. Fuerschbach ${ }^{1}$, William Kindel ${ }^{1}$, Patrick S. \\ Finnegan ${ }^{1}$, Joel R. Wendt ${ }^{1}$, Michael Gehl ${ }^{1}$, Hayden McGuinness ${ }^{1}$, Charles A. Walker ${ }^{1}$, \\ Anthony Lentine ${ }^{1}$, Shanalyn A. Kemme ${ }^{1}$, Grant Biedermann ${ }^{2}$, and Peter D. D. Schwindt ${ }^{1}$
}

${ }^{1}$ Sandia National Laboratories, Albuquerque, New Mexico 87185, USA

${ }^{2}$ Department of Physics and Astronomy, University of Oklahoma, Norman, Oklahoma 73019, USA

*jongmin.lee@sandia.gov

\begin{abstract}
The extreme miniaturization of a cold-atom interferometer accelerometer requires the development of novel technologies and architectures for the interferometer subsystems. We describe several component technologies and a laser system architecture to enable a path to such miniaturization. We developed a custom, compact titanium vacuum package containing a microfabricated grating chip for a tetrahedral grating magneto-optical trap (GMOT) using a single cooling beam. The vacuum package is integrated into the optomechanical design of a compact cold-atom sensor head with fixed optical components. In addition, a multichannel laser system driven by a single seed laser has been implemented with time-multiplexed frequency shifting using single sideband modulators, reducing the number of optical channels connected to the sensor head. This laser system architecture is compatible with a highly miniaturized photonic integrated circuit approach, and by demonstrating atom-interferometer operation with this laser system, we show feasibility for the integrated photonic approach. In the compact sensor head, sub-Doppler cooling in the GMOT produces $15 \mu \mathrm{K}$ temperatures, which can operate at a $20 \mathrm{~Hz}$ data rate for the atom interferometer sequence. After validating atomic coherence with Ramsey interferometry, we demonstrate a light-pulse atom interferometer in a gravimeter configuration without vibration isolation for $10 \mathrm{~Hz}$ measurement cycle rate and $T=0-4.5 \mathrm{~ms}$ interrogation time, resulting in $\Delta g / g=2.0 \times 10^{-6}$. All these efforts demonstrate progress towards deployable cold-atom inertial sensors under large amplitude motional dynamics.
\end{abstract}
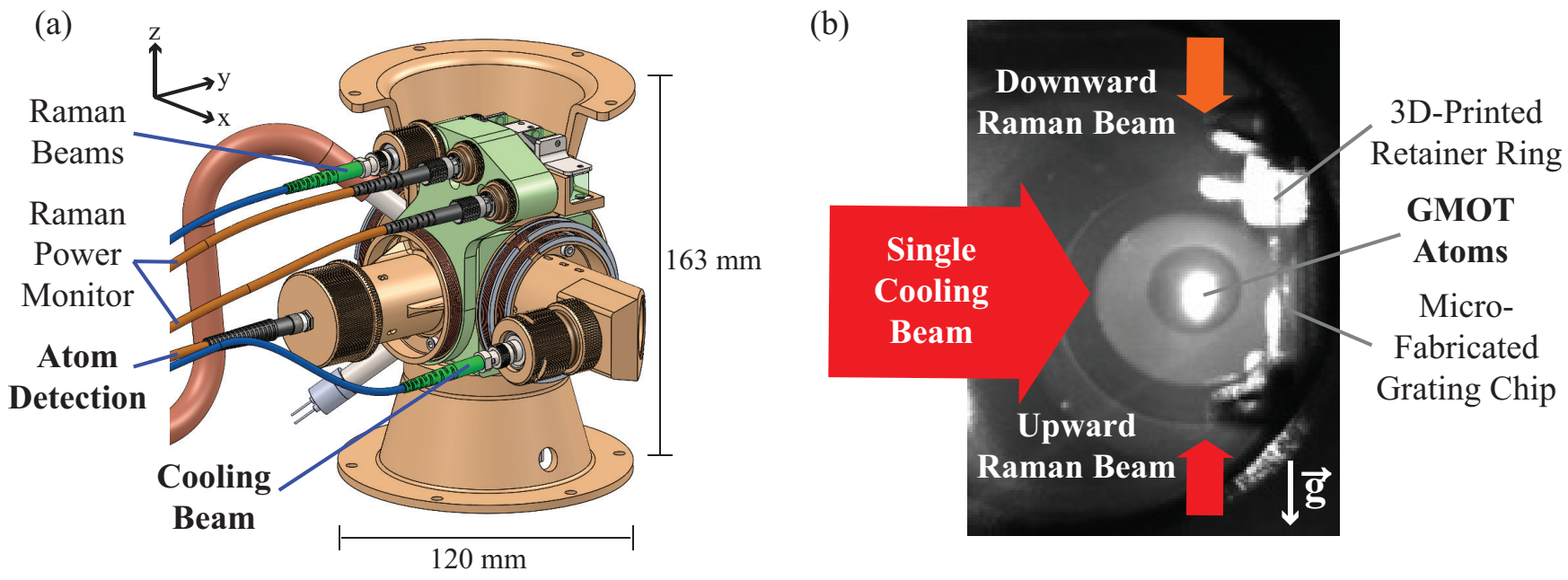

Figure 1. Concept of the compact light-pulse atom interferometer (LPAI) sensor head with fixed optical components and reliable optomechanical design for high dynamic conditions. (a) Conceptual 3D render of the compact LPAI sensor head. (b) Picture of the steady-state GMOT atoms in the sensor head. 


\section{Introduction}

Recently, there has been significant interest and investment made into quantum technologies for advanced sensing applications ${ }^{1-8}$. One area of particular interest is cold-atom inertial sensors based on a light-pulse atom interferometer (LPAI) ${ }^{9-15}$ using photon recoils from stimulated Raman transitions. The light pulse sequence of LPAIs, e.g., $\frac{\pi}{2} \rightarrow T \rightarrow \pi \rightarrow T \rightarrow \frac{\pi}{2}$, coherently splits, redirects, and recombines atomic wavepackets for matter wave interference, where $T$ is the interrogation time. During this process, the phase shifts experienced by the atoms are sensitive to inertial forces, resulting in an atomic interference fringe that provides acceleration and angular velocity information.

Over the past 30 years ${ }^{16,17}$, these LPAIs have demonstrated excellent laboratory sensitivity ${ }^{18}$ and serious efforts are underway to advance the platform for real-world applications ${ }^{19}$ and multi-axis sensors ${ }^{20-23}$. Their applications range from fundamental physics ${ }^{18,24,25}$ and geophysics ${ }^{26}$ to civil engineering ${ }^{27}$ and navigation, but bringing cold-atom sensors out of a well-controlled laboratory into a dynamic environment poses significant physics and engineering challenges that must be addressed. Recent mobile LPAI examples include land-based ${ }^{26,28}$, naval ${ }^{29}$, and airborne ${ }^{30-32}$ demonstrations under micro-g to $<2 \mathrm{~g}$ environments, where $\mathrm{g}$ is the acceleration due to gravity. Furthermore, Bose-Einstein condensates have been tested under micro-g in sounding rockets ${ }^{33}$ and an Earth-orbiting research laboratory ${ }^{34}$ with the aim of demonstrating LPAI functionality.

A significant challenge towards deployable cold-atom inertial sensors is achieving a compact form factor while maintaining high performance in extreme dynamics. A trade-off arises in LPAI operation because the highest sensitivity is achieved with long baseline atom interferometry capable of a long $T$, but high dynamics can move the atoms out of the interrogating laser beams and therefore demands a short interrogation time to mitigate those effects. In balancing this trade-off, a short time-of-flight approach ${ }^{35-37}$ produces an exceptional accelerometer that operates up to $330 \mathrm{~Hz}$. This was a first step toward potentially accommodating high-g environments (e.g., 1.5 to $9 \mathrm{~g}^{38-40}$ ) while maintaining high accelerometer performance (e.g., strategic-grade accelerometer: scale factor $<1 \mathrm{ppm}$, bias stability $<1 \mu \mathrm{g}^{41}$ ).

Here, we present progress on a multifaceted approach towards addressing the challenges of mobile LPAI operation and an initial demonstration of a cold atom inertial accelerometer based on a GMOT. Our LPAI sensor head is designed to perform counter-propagating Doppler-sensitive Raman without a retroreflecting mirror. This is in contrast to most LPAI systems $s^{26,28-30,32}$ that rely on a retroreflecting mirror to maintain the phase between two Raman beams, an approach that makes it difficult to distinguish oppositely diffracted atoms ${ }^{42}$. In particular, the sensor head without a retroreflecting mirror removes the degeneracy of low-velocity atoms present in retroreflecting Raman LPAIs ${ }^{42}$, enabling high data rate and operation of the sensor in any orientation.

With the objective of developing LPAIs for high-dynamic conditions, this necessarily subjects the laser system to similar dynamics and substantial efforts have been made towards developing compact and robust laser systems ${ }^{43-47}$ including the recent demonstration of a laser system with single sideband modulators (SSBMs) ${ }^{48,49}$. Many of these systems are based on discrete components that are connected through fiber-to-fiber connections or free-space optical paths with optomechanical alignment mounts, which limit their ability to withstand high dynamics and their ability to be mass-produced. Photonic integrated circuit (PIC) technology, based on microfabricated optical waveguides and components, offers the ability for robust laser systems that are scalable and readily mass produced ${ }^{50-57}$. Rubidium is particularly amenable to this approach because the optical resonance conveniently matches well with mature PIC technologies for light modulation ${ }^{50}$ and optical amplification at telecommunication wavelengths $(1560 \mathrm{~nm}$ ) before frequency doubling to $780 \mathrm{~nm}$ to address the atoms. In Section 3, we outline a PIC approach for a LPAI and demonstrate a laser system using commercial-off-the-shelf (COTS) components that realizes the essential function of the PIC-based architecture.

In this paper, we pursue efforts towards deployable cold-atom inertial sensors for real-world missions by reducing the size, weight and power (SWaP) of the LPAI sensor system, increasing the data rate of the LPAI operation for extended dynamic range, and utilizing laser and control systems robust against vibration, shock and radiation of a field test. The efforts include the development of microfabricated grating chips, custom Ti vacuum packages, a compact optomechanical design of the sensor head with fixed optical components, and a single $1560 \mathrm{~nm}$ seed laser system with time-multiplexed frequency shifting. Using the sensor head and the PIC-compatible laser system, we demonstrate a high data-rate sub-Doppler-cooled GMOT $(20 \mathrm{~Hz}$, $15 \mu \mathrm{K}, 1 \times 10^{6}$ atoms) with a single laser-cooling beam, atomic coherence with Ramsey interferometry, and proof-of-concept LPAI operation and measurement of gravity $\left(\Delta g / g=2.0 \times 10^{-6}, T=0-4.5 \mathrm{~ms}, 10 \mathrm{~Hz}\right)$ without vibration isolation.

\section{Compact Sensor Head}

The compact sensor head is designed as a tethered system with a remote electronics and laser system connected via cables and optical fibers (see Fig. 1(a)). The optomechanical design of the small form-factor sensor head supports a miniaturized Ti vacuum package and provides optical access for laser cooling and trapping, the Raman lasers, and fluorescence detection of the atoms. A GMOT ${ }^{58-64}$ is chosen for the production of cold ${ }^{87} \mathrm{Rb}$ atoms for the LPAI due to its simplified construction, elimination of unnecessary mechanical degrees-of-freedom, and superior optical access while only requiring a single cooling beam (see 
Fig. 1(b)). We developed an in-vacuum-mounted microfabricated reflective grating chip and demonstrated a tetrahedral GMOT with a single fiber-coupled optical channel.

\subsection{Grating Chip Fabrication}

For the compact LPAI sensor head, we designed micro-fabricated gratings to form the GMOT ${ }^{58-62}$, which uses a single incoming laser-cooling beam and multiple first-order diffracted beams. Our reflective grating chip is based on a triangular geometry with three 1-D binary grating sections generating a tetrahedral GMOT. Although the diffraction efficiency of binary gratings is lower than that of blazed and multilevel gratings, they are simpler to fabricate. First, we used Grating Diffraction Calculator ${ }^{65}$ to simulate a binary grating for the rubidium D2 transition at $780.24 \mathrm{~nm}^{66}$ for a variety of design parameters such as pitch, duty cycle, depth, polarization, and reflective surface material. We designed a binary grating etched with $\sim 50 \%$ duty-cycle and $195 \mathrm{~nm}$ depth. The diffraction efficiencies for $s$-polarized, $p$-polarized, and circularly polarized light, respectively, are 37.88 , 47.75 and $40.81 \%$ for the first order diffraction and $14.29,0.92$ and $7.61 \%$ for the zeroth order diffraction in the simulation. The first order diffraction angle is $40.56^{\circ}$ from the incoming beam, which is determined by $\theta_{\mathrm{m}}=\sin ^{-1}\left(\frac{\mathrm{m} \lambda}{\mathrm{d}}\right)$ for diffraction order $\mathrm{m}=1$, pitch $\mathrm{d}=1.2 \mu \mathrm{m}$, and wavelength $\lambda=780.24 \mathrm{~nm}$.

The fabrication process is shown in Fig. 2(b). A $195 \mathrm{~nm}$ layer of $\mathrm{SiO}_{2}$ was grown on a silicon wafer followed by spin-coating and soft-baking the E-beam resist (ER). The 1-D binary grating was then patterned in the ER by E-beam lithography. The pattern is etched into the $\mathrm{SiO}_{2}$ by using a selective reactive ion etching (RIE) process stopping on the silicon. The ER is then removed with solvents and all remaining organic residue is removed in an $\mathrm{O}_{2}$ plasma. A 50 nm reflective coating of aluminum $(\mathrm{Al})$ is deposited conformally over the etched $\mathrm{SiO}_{2}$ grating, using an angled and rotating platen in an E-beam evaporator. $\mathrm{Al}$ was chosen over Au due to the grating being inside vacuum where $\mathrm{Rb}$ can corrode $\mathrm{Au}^{67,68}$. The E-beam bias and the added sidewall thickness were carefully controlled to minimize errors in the target pitch, duty cycle, and depth of the grating.

The grating pattern is made in a circular shape $(17.5 \mathrm{~mm}$ diameter), which almost matches the fused-silica viewport ( $\sim 19 \mathrm{~mm}$ diameter) on the vacuum package. The grating chip is diced in a hexagonal shape where the non-grating flat edges are used for the retainer clips (see Fig. 2(a) and Fig. 3(b)).

\subsection{Titanium Vacuum Package}

The ultra-high vacuum (UHV) package is the centerpiece around which the sensor head is built. A photograph of the vacuum chamber contained in our sensor head is shown in Fig. 3(a). Briefly, the vacuum package is constructed from Grade-2 titanium (Ti) with five anti-reflection coated fused silica viewports, non-evaporable getters (SAES St172/HI/7.5-7), and alkali-metal dispensers (SAES Rb/NF/3.4/12FT10+10) housed inside Ti tubing laser-welded to the body. Ti has lower thermal expansion coefficient and a much lower hydrogen outgassing rate than stainless steel and is nonmagnetic. A copper exhaust port was also brazed to the body for evacuation and connection to an ion pump for testing purposes.

(a)

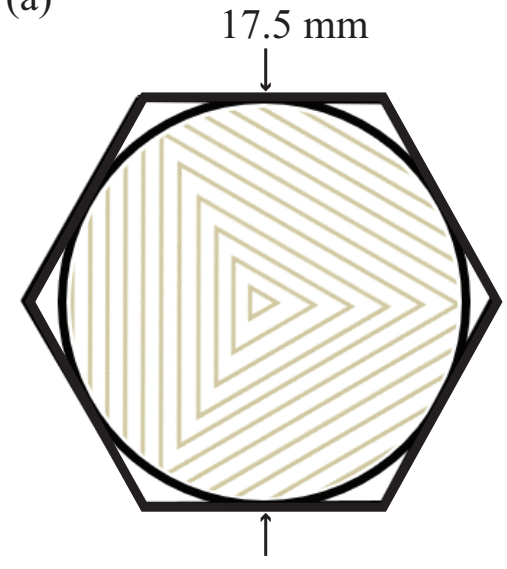

(b)

$\mathrm{SiO}_{2}$ deposition

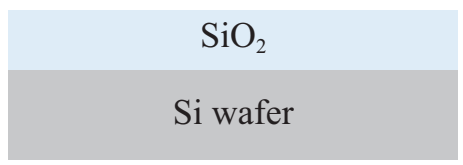

$\mathrm{SiO}_{2}$ etching and remove ER

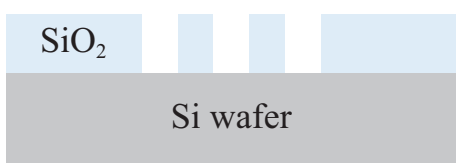

E-beam exposure and develop

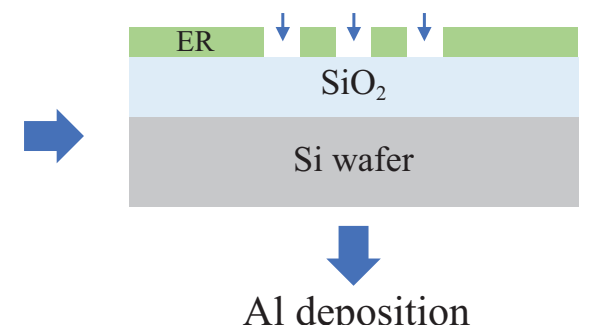

Al deposition

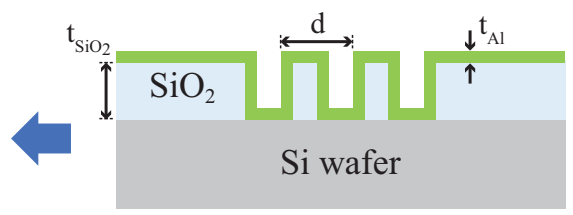

Figure 2. (a) A schematic of the hexagonal grating chip showing the side-to-side length of $17.5 \mathrm{~mm}$ and three grating areas symmetrically arranged in the circular shape. The non-grating flat edges are used for the grating retainer clips. The $1 / e^{2}$ beam diameter of the cooling beam is $28 \mathrm{~mm}$ truncated to $\approx 19 \mathrm{~mm}$ by the fused silica viewport. (b) Fabrication process of the GMOT grating chip. Parameters for the grating chip used in this paper are $\mathrm{t}_{\mathrm{Al}}=50 \mathrm{~nm}, \mathrm{t}_{\mathrm{SiO}_{2}}=195 \mathrm{~nm}, \mathrm{~d}=1.2 \mu \mathrm{m}$, and $\sim 50 \%$ duty cycle (details in main text). 
The GMOT grating chip is mounted internally and is held with a sintered metal 3D-printed Ti-alloy (ASTM Ti-6 Al-4 V) retainer having four swan-neck flexure mounts as shown in Fig. 3(b). The 3D-printed parts were vacuum baked at $450{ }^{\circ} \mathrm{C}$ prior to installing the grating chip. These flexure mounts provide optical access down to the surface of the grating chip for the Raman beams traveling parallel to the surface and avoids the use of epoxies.

The design of this vacuum package is similar in design to a passively pumped vacuum package ${ }^{69}$ that has now demonstrated more than a year of operation without active pumping. Passive pumping eliminates the need for an ion pump, reducing system complexity and SWaP. The passively pumped design in ${ }^{69}$ does not include the grating chip and uses sapphire windows (C-cut for low birefringence) for low helium permeation but future designs will include the grating chip.

\subsection{Compact LPAI Sensor Head with Fixed Optical Components}

For deployable cold-atom inertial sensors, we sought to eliminate most optomechanical alignment components in the design of the compact sensor head for operation in high vibration and harsh temperature conditions. In particular, the simplicity of a GMOT approach enables eliminating unnecessary mechanical degrees of freedom and achieves a compact and reliable optomechanical design. In addition, time-multiplexed frequency shifting of a single seed laser simplifies the required optical channels for the tethered sensor head.

The optomechanical structure supports the vacuum package and provides the necessary optical routing for a GMOT, atom detection, and Raman beams while reducing sensitivity to vibration. As shown in Fig. 4, the main structure surrounding the vacuum package primarily consists of a combination of FR4 fiberglass composite (green) and $30 \%$ glass-filled PEEK (brown) to reduce magnetic field interference due to eddy currents.

In Fig. 4(a), the horizontal cross-section view of the compact LPAI sensor head shows fixed optical components for cooling-beam and atom-detection channels for the GMOT. Using the grating chip (see Fig. 1(b) and Fig. 3(b)), a single incoming laser-cooling beam and three first-order diffracted beams creates a tetrahedral GMOT with a quadrupole magnetic field created by anti-Helmholtz coils. All four cooling beams and the magnetic field zero point have to be aligned to the same point in three-dimensional space. The final alignment of the cooling beam and the grating was accomplished through the 6-axis piezo stages which actively align the fiber-port-concave-lens assembly while monitoring the atomic cloud fluorescence via a board camera. After that, all the optics were bonded in place. In the GMOT, the estimated capture volume is $\approx 0.17 \mathrm{~cm}^{3}$. For steady-state GMOT atoms, the diameter of the atomic clouds is $\sim 0.8 \mathrm{~mm}$ perpendicular to the grating and $\sim 1.7 \mathrm{~mm}$ parallel to the grating, and the distance of the atomic clouds from the grating surface is $\sim 3.5 \mathrm{~mm}$. The repump/detection beam is coupled through the same fiber as the cooling beam with the same polarization.

In Fig. 4(b), the vertical cross-section view of the compact LPAI sensor head shows fixed optical components for crosslinearly-polarized Raman beams, Raman power monitor ports, and cooling beam channels. The Raman light is brought to the sensor head with a PM optical fiber where two Raman beams propagate along the fast and slow PM axes of the fiber.

(a)

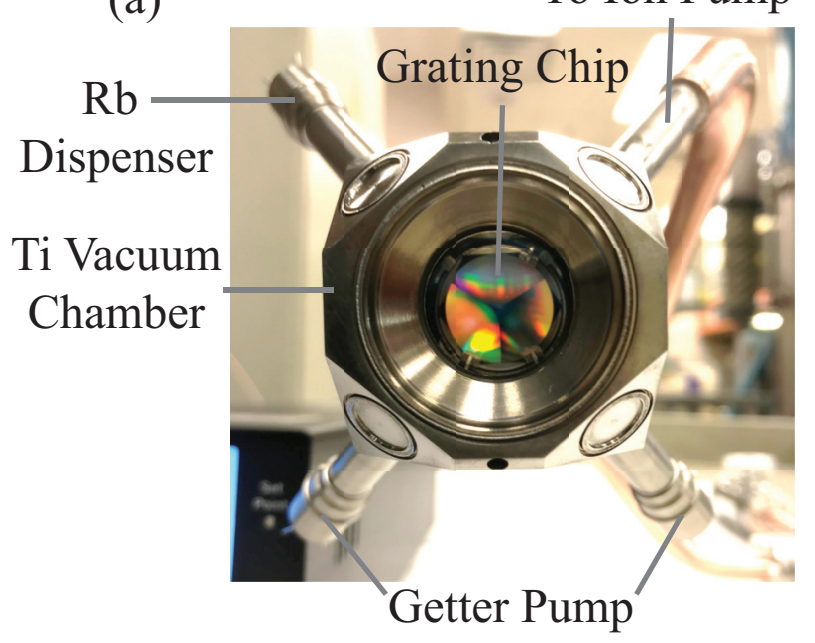

(b)

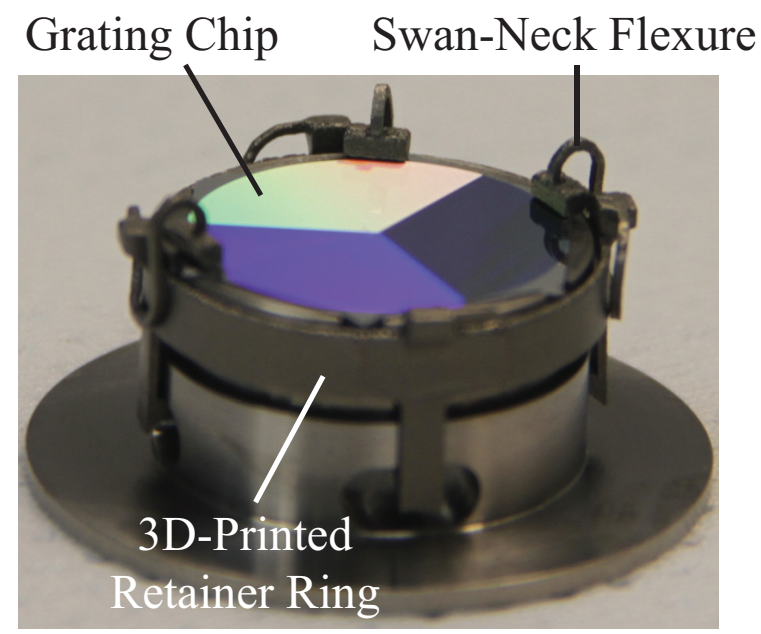

Figure 3. Images of the custom Ti vacuum package and the in-vacuum mounted grating chip. (a) View of the vacuum package looking at the grating chip through the GMOT cooling beam entry window $(\approx 19 \mathrm{~mm}$ clear aperture). The external dimensions of a truncated Ti cube are approximately $40.64 \times 40.64 \times 40.64 \mathrm{~mm}^{3}$. Also visible are Ti tubes which house the rubidium dispensers, getters, and a brazed connection to the $\mathrm{Cu}$ exhaust port. (b) Close-up view of the in-vacuum grating chip and mount prior to being laser-welded to the vacuum package. The grating chip is held in place with 3D-printed swan-neck flexure mounts. 
The Raman fiber launch assembly collimates the light, and a $45^{\circ}$-angled mirror brings the light into the plane of Fig. 4(b). After this, two Raman beams are split by the first PBS and become counter-propagating Doppler-sensitive Raman beams. The downward and upward Raman beams are applied to the atoms released from the GMOT, which drive state-dependent momentum kicks on the atoms for LPAI operation. The Raman power monitor ports couple light into multimode fibers such that the pulse amplitude of each Raman beam can be measured. Initial alignment was performed without the GMOT atoms using high-precision 6-axis piezo stages such that the Raman beams intersect at their designed position. However, we find in practice a mismatch between the optimized GMOT position and the designed Raman beam position. We note that the data shown below (Fig. 9(b) and Fig. 10) is taken by substituting the fixed Raman optics with conventional free space optics in order to optimize LPAI performance. This mismatch can be suppressed in subsequent versions of the sensor head and further reduced with active alignment or flat-top Raman beams to improve spatial-overlap homogeneity.

The detection system collects atomic fluorescence of GMOT atoms from a region which is a $\approx 3 \mathrm{~mm}$ diameter sphere where the atomic cloud exists during the LPAI operation. It consists of two focusing elements and a multimode collection fiber as shown in Fig. 4. The optics were centered in their housing using shims and bonded into place. A jam nut was tightened against the threaded interface while monitoring the atomic fluorescence from GMOT atoms with an avalanche photodiode (APD) module.

\section{Laser System with a Single Seed Laser and Time-Multiplexed Frequency Shifting}

A compact high-performance laser system is essential to reduce design complexity and improve reliability in dynamic environments. The long-term goal is to achieve extreme miniaturization of the laser system by integrating nearly all of the required optical components onto a single PIC. Over the last decade, silicon-based PICs operating in the telecom C-band have matured substantially, providing needed optical components for the LPAI application ${ }^{50}$ such as SSBMs for frequency shifting and variable optical attenuators (VOAs). With the Rb D2 line at $780 \mathrm{~nm}$, the optical frequency and power can be manipulated at $1560 \mathrm{~nm}$ using a PIC and then frequency doubled to $780 \mathrm{~nm}$. Additionally, there is ongoing work such as heterogeneously integrated silicon photonics and thin-film lithium niobate electro-optic modulators ${ }^{51,52}$, compound semiconductor and silicon photonics integration for a laser including semiconductor optical amplifiers (SOAs) ${ }^{53,54}$, silicon photonics with Brillouin amplifiers $^{55}$, and nonlinear photonics for second-harmonic generation (SHG) with lithium niobate ${ }^{56}$ and aluminum nitride ${ }^{57}$. Those on-going efforts would bring modulators, optical amplifiers, frequency doublers, and even laser sources onto the PIC for a fully integrated laser solution for an LPAI accelerometer. With chip-based technologies being highly scalable, moving from a single-axis accelerometer to a three-axis accelerometer or a complete LPAI-based inertial measurement unit is readily envisioned using PIC technology. Here, we outline a potential PIC-based architecture for the laser system and demonstrate a "PIC-compatible" laser system that shows the architecture is functional for an LPAI using discrete COTS components.

As shown in Fig. 5(a), our PIC-based laser system concept starts with an off-chip seed laser that is coupled onto a silicon PIC where a $1 \times N$ silicon nitride splitter divides the light into multiple channels. Within each channel, a SSBM is used to frequency offset the light to the required frequency for the particular function of the channel. With the SSBM, the frequency of the light can be rapidly switched between various frequencies (time-multiplexed frequency shifting) to allow a single channel to perform multiple functions, for example, repump light for the MOT and detection light for state read out (see Fig. 5 and Fig. 6). Our team has developed a multichannel SSBM PIC ${ }^{50}$ based on silicon photonics ${ }^{70}$, and we have performed an initial demonstration of using the SSBM PIC to generate the Raman lasers from the seed laser in an LPAI, which will be reported in a later publication. To control the amplitude of light, a variable optical attenuator (VOA) is implemented in each channel of silicon photonics before the output of the PIC device. Our team has efforts to develop the high-power multi-stage SOAs and high-efficiency frequency doublers. Subsequent to the SSBM, the light would go into a $1560 \mathrm{~nm}$ high-power compound semiconductor optical amplifier (SOA) producing roughly $500 \mathrm{~mW}$ followed by a frequency doubler. This SOA can also operate as a high-speed (sub-nanosecond) optical switch ${ }^{71}$ with a high extinction ratio. Using a lithium niobate platform with shallow etched waveguides ${ }^{72}, 939 \% \mathrm{~W}^{-1}$ SHG conversion efficiency has been demonstrated. While Fig. 5(a) shows separate PIC chips for silicon photonics, compound semiconductor, and nonlinear photonics, we envision a future PIC where all required optical components are hybrid integrated into a single PIC.

The PIC-compatible laser system is shown in Fig. 5(b), where essential functions of the PIC architecture are replaced with fiber-connected commercial-off-the-shelf (COTS) components. A single $1560 \mathrm{~nm}$ fiber seed laser with $\leq 3 \mathrm{kHz}$ linewidth (NP Photonics FLM-150-3-1560.49-1-S-A) is split into five channels: (1) Lock, (2) Cooling, (3) Repump/Detection, (4) Raman 1, and (5) Raman 2. Channels 1, 2, 3 share similar architectures with light from the seed laser being shifted by the $\mathrm{SSBM}^{48,49}$ (IQ modulator, EO Space IQ-0D6S-25-PFA-PFA-LV). The Lock channel has an additional phase modulator $\left(\Phi_{\mathrm{MOD}}, \mathrm{EO}\right.$ Space PM-0S5-10-PFA-PFA-UL) after the SSBM for stabilizing the frequency of the seed laser to a rubidium reference $\left(|F=2\rangle \rightarrow\left|F^{\prime}=2,3\right\rangle\right.$ crossover resonance in $\left.{ }^{87} \mathrm{Rb}\right)$ with frequency modulation saturation spectroscopy. The $1560 \mathrm{~nm}$ light is then amplified by an erbium-doped fiber amplifier (EDFA, IPG Photonics EAR-2K-C-LP-SF) and passes through a high-power electro-optic VOA (Agiltron NHOA-325115333) and a high-power electro-optic optical switch (SW, Agiltron 
NHSW-115115333) before frequency doubling to $780 \mathrm{~nm}$ with the fiber-SHG modules (SHG, AdvR RSH-T0780-P15P78AL0).

The Cooling channel provides the cooling light on the $|F=2\rangle \rightarrow\left|F^{\prime}=3\right\rangle$ transition with the intensity of about $1.1 \mathrm{~mW} \mathrm{~cm}^{-2}$ and the detuning of $-0.5 \Gamma$ for steady-state GMOT operation (see Fig. 6 (a)), where $\Gamma / 2 \pi=6.065 \mathrm{MHz}$ for ${ }^{87} \mathrm{Rb} \mathrm{D} 2$ transition. After the GMOT is loaded, sub-Doppler cooling is accomplished with a frequency ramp from $-0.5 \Gamma$ to $-13.5 \Gamma$ and a VOA-controlled intensity ramp (Fig. 6(b)). A $15 \mu \mathrm{K}$ temperature of sub-Doppler cooled GMOT atoms is determined from the width of the Doppler-sensitive Raman resonance. After sub-Doppler cooling, the repump light is extinguished and the Cooling channel jumps to the $|F=2\rangle \rightarrow\left|F^{\prime}=2\right\rangle$ transition to depump the atoms into $|F=1\rangle$ hyperfine ground state (Fig. 6(b)).

For fluorescence detection of atoms, the Repump/Detection channel is first resonant with the $|F=2\rangle \rightarrow\left|F^{\prime}=3\right\rangle$ transition to detect atoms in the $|F=2\rangle$ state (see Fig. 6(a)). It then jumps to $|F=1\rangle \rightarrow\left|F^{\prime}=2\right\rangle$ to repump all atoms into the $|F=2\rangle$ state before jumping back to $|F=2\rangle \rightarrow\left|F^{\prime}=3\right\rangle$ transition to detect all the atoms (Fig. 6 (b)). In the experiment, the Repump/Detection channel is coupled to the same fiber for the Cooling channel with the same polarization.

For the Raman channels, time-multiplexed frequency shifting can also be implemented but was not pursued here in order to simplify phase stabilization. The cross-linearly-polarized Raman beams control the internal states of atoms, and in particular Doppler-sensitive Raman beams provide the state-dependent momentum kicks to measure inertial forces on the atoms. Both Raman frequencies are derived from the same $1560 \mathrm{~nm}$ seed laser as shown in Fig. 5(b). The Raman 1 is the frequency-doubled seed laser $(780 \mathrm{~nm})$ that is $\sim 1.15 \mathrm{GHz}$ red-detuned from the $|F=2\rangle \rightarrow\left|F^{\prime}=3\right\rangle$ resonance. Raman 2 acquires a frequency offset via a fiber-coupled $1560 \mathrm{~nm}$ acousto optic modulator (AOM) operating at $-150 \mathrm{MHz}$. Then, the frequency-offset light is amplified by a $1560 \mathrm{~nm}$ SOA and frequency-doubled to $780 \mathrm{~nm}$. After that, Raman 2 is phase-modulated by a $780 \mathrm{~nm}$ phase modulator followed by amplification by a $780 \mathrm{~nm}$ SOA.

Both Raman beams are combined with orthogonal polarization using free-space optics as shown in Fig. 7. A sample of the combined beams $(\sim 10 \%)$ is used for continuous Raman phase stabilization through the fiber-coupled $1560 \mathrm{~nm}$ AOM and a voltage-controlled oscillator while the majority of the power is sent to a free-space AOM for optical switching. The combined Raman light is then coupled to a single PM fiber along its two birefringent axes for delivery to the LPAI sensor head, reducing noise from varying paths. The Raman spectroscopy has been applied to Ramsey interferometry with co-propagating Doppler-free Raman beams (Fig. 9(b)) and atom interferometry with counter-propagating Doppler-sensitive Raman beams (Fig. 10(c)). This is based on stimulated Raman transitions (Fig. 10(a)) composed of two light frequency components such as Raman 1 and Raman 2.

\section{Experimental Demonstration}

As a step towards deployable cold-atom inertial sensors, the compact sensor head (Section 2) and the PIC-compatible laser system (Section 3) have been developed. Based on these components, we demonstrate a high data-rate sub-Doppler-cooled GMOT with a single laser-cooling beam, validate atomic coherence with Ramsey interferometry, and perform proof-of-concept LPAI operation and measurement of gravity.

\subsection{High Data-Rate GMOT Operation}

High data-rate operation enhances the dynamic range of LPAIs by mitigating detrimental effects of sensor head motion during operation. Efficient atom recapture is critical to maintaining a reasonably high atom number during high data-rate operation. Previous studies ${ }^{35,36}$ showed the potential of high data-rate LPAI operation (up to $330 \mathrm{~Hz}$ for cold atoms) that can be operable in a dynamic environment while maintaining good sensitivity. High data-rate LPAI operation in our system follows this sequence: (1) capture and cool atoms from background vapor with a GMOT, (2) further laser-cool the atoms with sub-Doppler cooling, (3) prepare the atoms in the $|F=1\rangle$ state, (4) release to free space, (5) perform physics experiments, (6) detect the atomic populations, and (7) recapture atoms for the next measurement cycle.

Here we demonstrate proof-of-concept high data-rate GMOT operation by measuring the sub-Doppler cooled atom number vs. data rate (see Fig. 8(a-b)). For this measurement, the atom number was determined by absorption of a probe beam resonant with the $|F=2\rangle \rightarrow\left|F^{\prime}=3\right\rangle$ transition. Within a measurement cycle, the MOT is loaded for a variable amount of time before the quadrupole magnetic field is turned off. Sub-Doppler cooling and depumping to $|F=1\rangle$ occurs over the next $3 \mathrm{~ms}$ and is followed by a $13 \mathrm{~ms}$ window where physics experiments and state detection occur. At the end of the window, the cooling light and quadrupole field are turned back on to recapture atoms and restart MOT loading. The data rate is the inverse of the total cycle time. The number of sub-Doppler-cooled GMOT atoms decreases as the data rate increases as shown in Fig. 8(a), and the same data is replotted in Fig. 8(b) as the MOT loading time increases. The current data-rate measurement of GMOT operation is limited by the data rate of the control system and the low recapture efficiency likely due to the low GMOT cooling intensity ${ }^{73}$.

\subsection{Ramsey Interferometry with a GMOT}

We examine atomic coherence to show that the system is not limited by decoherence due to background collisions/pressure and spatial-overlap inhomogeneity between cold atoms and Raman beams. We observe Rabi oscillations and Ramsey fringes of 
the GMOT atoms by using both a microwave field and co-propagating Doppler-free Raman beams that address the "clock" transition in the hyperfine ground state manifold, i.e., $\left|F=1, m_{F}=0\right\rangle$ to $\left|F=2, m_{F}=0\right\rangle$.

In microwave Rabi measurement, the Rabi frequency was $10 \mathrm{kHz}\left(\mathrm{T}_{2 \pi}=100 \mu \mathrm{s}\right)$, and the Rabi coherence time was $\tau_{1 / e} \simeq$ $676 \mu$ s. In Doppler-free-Raman Rabi measurement, the Rabi frequency was $130 \mathrm{kHz}\left(\mathrm{T}_{2 \pi}=7.68 \mu \mathrm{s}\right)$ and the Rabi coherence time was $\tau_{1 / e} \simeq 8.3 \mu \mathrm{s}$. The measured pulse times of the Rabi measurements were used for the Ramsey measurements.

For the study of Ramsey interferometry $\left(\frac{\pi}{2} \rightarrow T \rightarrow \frac{\pi}{2}\right)$, frequency detuning of the two $\frac{\pi}{2}$-pulses has been scanned for a fixed time $T$ to measure atomic interference fringes based on the two hyperfine ground states as shown in Fig. 9. In microwave Ramsey measurement, the fringe spacing was $2.27 \mathrm{kHz}$ from the data fitting, which is consistent with $1 / T$ for $T=450 \mu$ s (see Fig. 9(a)). In Doppler-free-Raman Ramsey measurement, the fringe spacing was $19.74 \mathrm{kHz}$ from the data fitting, which also corresponds to $1 / T$ for $T=48.08 \mu$ s (see Fig. 9(b)).

The improvement of spatial-overlap homogeneity between the GMOT atoms and the Raman beams becomes important to achieve the best performance in Ramsey interferometry and atom interferometry ${ }^{74}$. The beam diameter of the Raman beams is $\mathrm{D}_{1 / \mathrm{e}^{2}, \text { Raman }} \simeq 5.4 \mathrm{~mm}$, and the diameter of the atomic clouds parallel to the grating is $\sim 1.7 \mathrm{~mm}$ comparable to one third of the $\mathrm{D}_{1 / \mathrm{e}^{2}, \text { Raman }}$. The spatial-overlap homogeneity can be further enhanced with flat-top Raman beams ${ }^{75}$ or large Raman beams ${ }^{75}$.

\subsection{Light-Pulse Atom Interferometry with a GMOT}

We demonstrate proof-of-concept LPAI operation and gravity measurement using a GMOT. This integrates all of our efforts: microfabricated grating chips, custom Ti vacuum packages, a compact sensor head with fixed optical components, and a single seed laser system with time-multiplexed frequency shifting. Our multifaceted approach can suggest a solution to the challenges of realizing a compact LPAI inertial sensor in high dynamics while maintaining the performance by reducing the SWaP of the sensor system, increasing the data rate of the LPAI to extend the dynamic range, and utilizing reliable and agile laser and control systems. In our LPAIs, lin-perp-lin polarization has been used for counter-propagating Doppler-sensitive Raman without a retroreflecting mirror, which can remove the degeneracy of low-velocity atoms and enable high data rate and operation of the sensor in any orientation.

In a gravimeter configuration, the LPAI accelerometer operates using stimulated Raman transitions (Fig. 10(a)), which coherently addresses the two hyperfine ground states of atoms, i.e., $\left|F=1, m_{F}=0\right\rangle$ and $\left|F=2, m_{F}=0\right\rangle$, and drives photon recoils on the atoms along the direction of gravity using counter-propagating Raman beams. The three-light-pulse sequence of the LPAI coherently splits, redirects, and recombines atomic wavepackets for atomic interference in space-time trajectory (Fig. 10(b)). The atomic interference fringe is sensitive to inertial forces that the atoms experience during the LPAI pulse sequence, and the phase shift provides gravitational acceleration information.

As discussed in Section 2.3, we optimize spatial overlap between the GMOT atoms and the Raman beams using free-space optomechanical components around the sensor head for Raman beams instead of the fully fixed optical components shown in Fig. 4(b). The sensor head assembly was located on a counter top without any vibration isolation. The LPAI accelerometer measures the gravitational acceleration by the phase shift of atomic interference fringe, i.e., $\Delta \phi_{\mathrm{acc}}=k_{\mathrm{eff}} \cdot a T^{2}$. When the sensing axis of Doppler-sensitive Raman beams is aligned to the gravity direction $(a=g)$. The Raman quantization axis was defined along the Z-axis with a magnetic field of $1.34 \mathrm{G}$, and the sensor head was located inside a single-layer magnetic shield. Since the velocity of the atoms increases linearly due to $g$, the LPAI fringe becomes chirped as the interrogation time $T$ is increased. Based on the fit of a chirped sinusoid ${ }^{35}$, we measured $g=9.79316(2) \mathrm{m} \mathrm{s}^{-2}$ with LPAI operation up to $T=4.5 \mathrm{~ms}$ as shown in Fig. 10(c-e). The statistical uncertainty is $\Delta g / g=2.0 \times 10^{-6}$ from fitting the data from $T=0.0-4.5 \mathrm{~ms}$.

\section{Conclusion}

We demonstrate significant scientific and engineering progress toward developing deployable cold-atom inertial sensors that can maintain excellent inertial sensitivity in a dynamic environment. Our compact cold-atom sensor head uses fixed optical components, a custom vacuum package, and a custom grating for a GMOT with the aim of increased ruggedization and miniaturization of the system. Using a miniaturized fixed-optics assembly constructed with rigid materials is essential for operation in dynamic environments. While our initial attempt at using fixed optics for the Raman beams was unsuccessful, we expect active alignment of the optics while monitoring the atomic signal will ultimately allow us to fix the Raman beam optics in place. We also introduced a PIC-based architecture for the LPAI laser system with a single $1560 \mathrm{~nm}$ seed laser and time-multiplexed frequency shifting by single sideband modulators. With the sensor head and the PIC-compatible laser system, we demonstrated a high data-rate sub-Doppler-cooled GMOT $\left(20 \mathrm{~Hz}, 15 \mu \mathrm{K}, 1 \times 10^{6}\right.$ atoms $)$ using a single laser-cooling beam and a microfabricated grating chip. After validating atomic coherence with Ramsey interferometry, we demonstrate proof-of-concept LPAI operation and measurement of gravity $\left(\Delta g / g=2.0 \times 10^{-6}, T=0-4.5 \mathrm{~ms}, 10 \mathrm{~Hz}\right)$ without vibration isolation. These efforts show the potential for compact, multi-axis, and deployable cold-atom inertial sensors with microfabricated grating chips. In addition, the PIC-compatible laser architecture validates functional operation of a PIC-based 
laser system composed of silicon photonics for single sideband modulation, compound semiconductor for optical amplification, and nonlinear photonics for frequency doubling towards a co-packaged laser system and ultimately a single hybrid-integrated PIC laser system. Simultaneously, our team has developed those PIC components for deployable cold-atom inertial sensors. We anticipate that these efforts will significantly decrease the cost and reduce manufacturing and testing time in realizing a complete matter wave inertial measurement unit, i.e., three-axis atomic accelerometers and three-axis atomic gyroscopes, which will have tens of optical channels. The technologies developed in these on-going efforts can also be applied beyond LPAI gravimeters, gravity gradiometers, accelerometers, and gyroscopes to other real-world atomic sensor applications, such as clocks, magnetometers, and electric field sensors.

\section{Funding}

This work was supported by the Laboratory Directed Research and Development program at Sandia National Laboratories.

\section{Acknowledgments}

We thank Daniel Soh, Connor Brasher, Joseph Berg, Tony G. Smith, Greg Hoth, Bethany Little, Dennis J. De Smet, Melissa Revelle, Ashok Kodigala, Christopher T. DeRose, Erik J. Skogen, Allen Vawter, Matt Eichenfield, Aleem Siddiqui, Peter Rakich, and Shayan Mookherjea for their support and helpful discussion. Sandia National Laboratories is a multimission laboratory managed and operated by National Technology \& Engineering Solutions of Sandia, LLC, a wholly owned subsidiary of Honeywell International Inc., for the U.S. Department of Energy's National Nuclear Security Administration under contract DE-NA0003525. This paper describes objective technical results and analysis. Any subjective views or opinions that might be expressed in the paper do not necessarily represent the views of the U.S. Department of Energy or the United States Government.

\section{Disclosures}

The authors declare no conflicts of interest.

\section{References}

1. Heavner, T. P. et al. First accuracy evaluation of NIST-F2. Metrologia 51, 174-182, DOI: 10.1088/0026-1394/51/3/174 (2014).

2. Wynands, R. \& Weyers, S. Atomic fountain clocks. Metrologia 42, S64-S79, DOI: 10.1088/0026-1394/42/3/s08 (2005).

3. Nicholson, T. L. et al. Systematic evaluation of an atomic clock at $2 \times 10^{-18}$ total uncertainty. Nat. Commun. 6, 1, DOI: 10.1038/ncomms7896 (2015).

4. Ludlow, A. D., Boyd, M. M., Ye, J., Peik, E. \& Schmidt, P. O. Optical atomic clocks. Rev. Mod. Phys. 87, 637-701, DOI: 10.1103/RevModPhys.87.637 (2015).

5. Dang, H. B., Maloof, A. C. \& Romalis, M. V. Ultrahigh sensitivity magnetic field and magnetization measurements with an atomic magnetometer. Appl. Phys. Lett. 97, 151110, DOI: 10.1063/1.3491215 (2010).

6. Holloway, C. L. et al. Broadband Rydberg Atom-Based Electric-Field Probe for SI-Traceable, Self-Calibrated Measurements. IEEE Transactions on Antennas Propag. 62, 6169-6182, DOI: 10.1109/TAP.2014.2360208 (2014).

7. Johnson, C., Schwindt, P. D. D. \& Weisend, M. Magnetoencephalography with a two-color pump-probe, fiber-coupled atomic magnetometer. Appl. Phys. Lett. 97, 243703, DOI: 10.1063/1.3522648 (2010).

8. Grujic, Z., Koss, P. A., Bison, G. \& Weis, A. A sensitive and accurate atomic magnetometer based on free spin precession. The Eur. Phys. J. D 69, DOI: 10.1140/epjd/e2015-50875-3 (2015).

9. Kasevich, M. \& Chu, S. Atomic interferometry using stimulated Raman transitions. Phys. Rev. Lett. 67, 181-184, DOI: 10.1103/PhysRevLett.67.181 (1991).

10. Gustavson, T. L., Bouyer, P. \& Kasevich, M. A. Precision Rotation Measurements with an Atom Interferometer Gyroscope. Phys. Rev. Lett. 78, 2046-2049, DOI: 10.1103/PhysRevLett.78.2046 (1997).

11. Peters, A., Chung, K. Y. \& Chu, S. Measurement of gravitational acceleration by dropping atoms. Nature 400, 849-852, DOI: 10.1038/23655 (1999).

12. McGuirk, J. M., Foster, G. T., Fixler, J. B., Snadden, M. J. \& Kasevich, M. A. Sensitive absolute-gravity gradiometry using atom interferometry. Phys. Rev. A 65, 033608, DOI: 10.1103/PhysRevA.65.033608 (2002). 
13. Cronin, A. D., Schmiedmayer, J. \& Pritchard, D. E. Optics and interferometry with atoms and molecules. Rev. Mod. Phys. 81, 1051-1129, DOI: 10.1103/RevModPhys.81.1051 (2009).

14. Kovachy, T. et al. Quantum superposition at the half-metre scale. Nature 528, 530-533, DOI: 10.1038/nature16155 (2015).

15. Parker, R. H., Yu, C., Zhong, W., Estey, B. \& Müller, H. Measurement of the fine-structure constant as a test of the Standard Model. Science 360, 191-195, DOI: 10.1126/science.aap7706 (2018).

16. Geiger, R., Landragin, A., Merlet, S. \& Pereira Dos Santos, F. High-accuracy inertial measurements with cold-atom sensors. AVS Quantum Sci. 2, 024702, DOI: 10.1116/5.0009093 (2020).

17. Tino, G. M. Testing gravity with cold atom interferometry: results and prospects. Quantum Sci. Technol. 6, 024014, DOI: 10.1088/2058-9565/abd83e (2021).

18. Asenbaum, P., Overstreet, C., Kim, M., Curti, J. \& Kasevich, M. A. Atom-interferometric test of the equivalence principle at the $10^{-12}$ level. Phys. Rev. Lett. 125, 191101, DOI: 10.1103/PhysRevLett.125.191101 (2020).

19. Bongs, K. et al. Taking atom interferometric quantum sensors from the laboratory to real-world applications. Nat. Rev. Phys. 1, 731-739, DOI: 10.1038/s42254-019-0117-4 (2019).

20. Canuel, B. et al. Six-axis inertial sensor using cold-atom interferometry. Phys. Rev. Lett. 97, 010402, DOI: 10.1103/ PhysRevLett.97.010402 (2006).

21. Hardman, K. S. et al. Simultaneous precision gravimetry and magnetic gradiometry with a bose-einstein condensate: A high precision, quantum sensor. Phys. Rev. Lett. 117, 138501, DOI: 10.1103/PhysRevLett.117.138501 (2016).

22. Wu, X. et al. Multiaxis atom interferometry with a single-diode laser and a pyramidal magneto-optical trap. Optica 4, 1545-1551, DOI: 10.1364/OPTICA.4.001545 (2017).

23. Chen, Y.-J. et al. Single-source multiaxis cold-atom interferometer in a centimeter-scale cell. Phys. Rev. Appl. 12, 014019, DOI: 10.1103/PhysRevApplied.12.014019 (2019).

24. Rosi, G., Sorrentino, F., Cacciapuoti, L., Prevedelli, M. \& Tino, G. M. Precision measurement of the Newtonian gravitational constant using cold atoms. Nature 510, 518-521, DOI: 10.1038/nature13433 (2014).

25. Canuel, B. et al. Exploring gravity with the MIGA large scale atom interferometer. Sci. Reports 8, DOI: 10.1038/ s41598-018-32165-z (2018).

26. Wu, X. et al. Gravity surveys using a mobile atom interferometer. Sci. Adv. 5, DOI: 10.1126/sciadv.aax0800 (2019).

27. Hinton, A. et al. A portable magneto-optical trap with prospects for atom interferometry in civil engineering. Philos. Transactions Royal Soc. A: Math. Phys. Eng. Sci. 375, 20160238, DOI: 10.1098/rsta.2016.0238 (2017).

28. Ménoret, V. et al. Gravity measurements below $10^{-9} \mathrm{~g}$ with a transportable absolute quantum gravimeter. Sci. Reports $\mathbf{8}$, DOI: 10.1038/s41598-018-30608-1 (2018).

29. Bidel, Y. et al. Absolute marine gravimetry with matter-wave interferometry. Nat. Commun. 9, 627, DOI: 10.1038/ s41467-018-03040-2 (2018).

30. Geiger, R. et al. Detecting inertial effects with airborne matter-wave interferometry. Nat. Commun. 2, DOI: 10.1038/ ncomms1479 (2011).

31. Barrett, B. et al. Dual matter-wave inertial sensors in weightlessness. Nat. Commun. 7, DOI: 10.1038/ncomms 13786 (2016).

32. Bidel, Y. et al. Absolute airborne gravimetry with a cold atom sensor. J. Geod. 94, 20, DOI: 10.1007/s00190-020-01350-2 (2020).

33. Becker, D. et al. Space-borne Bose-Einstein condensation for precision interferometry. Nature 562, 391-395, DOI: 10.1038/s41586-018-0605-1 (2018).

34. Aveline, D. C. et al. Observation of Bose-Einstein condensates in an Earth-orbiting research lab. Nature 582, 193-197, DOI: $10.1038 / \mathrm{s} 41586-020-2346-1$ (2020).

35. McGuinness, H. J., Rakholia, A. V. \& Biedermann, G. W. High data-rate atom interferometer for measuring acceleration. Appl. Phys. Lett. 100, 011106, DOI: 10.1063/1.3673845 (2012).

36. Rakholia, A. V., McGuinness, H. J. \& Biedermann, G. W. Dual-Axis High-Data-Rate Atom Interferometer via Cold Ensemble Exchange. Phys. Rev. Appl. 2, 054012, DOI: 10.1103/PhysRevApplied.2.054012 (2014). 
37. Adams, B. et al. The development of a High data rate atom interferometric gravimeter (HIDRAG) for gravity map matching navigation. In 2021 IEEE International Symposium on Inertial Sensors and Systems (INERTIAL), 1-4, DOI: 10.1109/INERTIAL51137.2021.9430461 (2021).

38. Nagy, J. A. \& Jr., G. L. C. Flight Vibration Data of the Aerobee 150A Sounding Rocket. Tech. Rep., NASA (1964).

39. Schoenster, J. A. Measurements and Analysis of Solid-Propellant-Rocket Vibrations Obtained during a Captive Flight. Tech. Rep., NASA (1971).

40. Office, S. R. P. NASA Sounding Rockets User Handbook. Tech. Rep., NASA (2015).

41. Travagnin, M. Cold atom interferometry for inertial navigation sensors. technology assessment: space and defence applications. Tech. Rep., Joint Research Centre (2020). DOI: 10.2760/237221.

42. Perrin, I. et al. Zero-velocity atom interferometry using a retroreflected frequency-chirped laser. Phys. Rev. A 100, 053618, DOI: 10.1103/PhysRevA.100.053618 (2019).

43. Lévèque, T., Antoni-Micollier, L., Faure, B. \& Berthon, J. A laser setup for rubidium cooling dedicated to space applications. Appl. Phys. B 116, 997-1004, DOI: 10.1007/s00340-014-5788-z (2014).

44. Schkolnik, V. et al. A compact and robust diode laser system for atom interferometry on a sounding rocket. Appl. Phys. B 122, DOI: 10.1007/s00340-016-6490-0 (2016).

45. Caldani, R. et al. A prototype industrial laser system for cold atom inertial sensing in space. The Eur. Phys. J. D 73, DOI: 10.1140/epjd/e2019-100360-2 (2019).

46. Sabulsky, D. O. et al. A fibered laser system for the MIGA large scale atom interferometer. Sci. Reports 10, DOI: 10.1038/s41598-020-59971-8 (2020).

47. Frye, K. et al. The Bose-Einstein Condensate and Cold Atom Laboratory. EPJ Quantum Technol. 8, DOI: 10.1140/epjqt/ s40507-020-00090-8 (2021).

48. Zhu, L. et al. Application of optical single-sideband laser in Raman atom interferometry. Opt. Express 26, 6542-6553, DOI: 10.1364/OE.26.006542 (2018).

49. Rammeloo, C., Zhu, L., Lien, Y.-H., Bongs, K. \& Holynski, M. Performance of an optical single-sideband laser system for atom interferometry. J. Opt. Soc. Am. B 37, 1485-1493, DOI: 10.1364/JOSAB.385919 (2020).

50. Kodigala, A. et al. Silicon Photonic Single-Sideband Generation with Dual-Parallel Mach-Zehnder Modulators. In Conference on Lasers and Electro-Optics, STh4N.6, DOI: 10.1364/CLEO_SI.2019.STh4N.6 (Optical Society of America, 2019).

51. Weigel, P. O. et al. Bonded thin film lithium niobate modulator on a silicon photonics platform exceeding $100 \mathrm{GHz} 3-\mathrm{dB}$ electrical modulation bandwidth. Opt. Express 26, 23728-23739, DOI: 10.1364/OE.26.023728 (2018).

52. Boynton, N. et al. A heterogeneously integrated silicon photonic/lithium niobate travelling wave electro-optic modulator. Opt. Express 28, 1868-1884, DOI: 10.1364/OE.28.001868 (2020).

53. Heck, M. J. R. et al. Hybrid silicon photonics for optical interconnects. IEEE J. Sel. Top. Quantum Electron. 17, 333-346, DOI: 10.1109/JSTQE.2010.2051798 (2011).

54. Davis, J. A. et al. III/V silicon hybrid laser based on a resonant Bragg structure. Appl. Opt. 59, 4158-4164, DOI: 10.1364/AO.390522 (2020).

55. Otterstrom, N. T. et al. Resonantly enhanced nonreciprocal silicon Brillouin amplifier. Optica 6, 1117-1123, DOI: 10.1364/OPTICA.6.001117 (2019).

56. Moore, J. et al. Efficient Second Harmonic Generation in Lithium Niobate on Insulator. In Conference on Lasers and Electro-Optics, STh3P.1, DOI: 10.1364/CLEO_SI.2016.STh3P.1 (Optical Society of America, 2016).

57. Siddiqui, A. M. et al. Optimization of Si-Photonics Compatible AlN waveguides for Integrated Nonlinear Optics Applications. In Conference on Lasers and Electro-Optics, JTu2A.96, DOI: 10.1364/CLEO_AT.2019.JTu2A.96 (Optical Society of America, 2019).

58. Vangeleyn, M., Griffin, P. F., Riis, E. \& Arnold, A. S. Laser cooling with a single laser beam and a planar diffractor. Opt. Lett. 35, 3453-3455, DOI: 10.1364/OL.35.003453 (2010).

59. Nshii, C. C. et al. A surface-patterned chip as a strong source of ultracold atoms for quantum technologies. Nat. Nanotechnol. 8, 321-324, DOI: 10.1038/nnano.2013.47 (2013). 
60. Lee, J., Grover, J. A., Orozco, L. A. \& Rolston, S. L. Sub-Doppler cooling of neutral atoms in a grating magneto-optical trap. J. Opt. Soc. Am. B 30, 2869-2874, DOI: 10.1364/JOSAB.30.002869 (2013).

61. Imhof, E. et al. Two-dimensional grating magneto-optical trap. Phys. Rev. A 96, 033636, DOI: 10.1103/PhysRevA.96. 033636 (2017).

62. McGilligan, J. P. et al. Grating chips for quantum technologies. Sci. Reports 7, DOI: 10.1038/s41598-017-00254-0 (2017).

63. Barker, D. S. et al. Single-Beam Zeeman Slower and Magneto-Optical Trap Using a Nanofabricated Grating. Phys. Rev. Appl. 11, 064023, DOI: 10.1103/PhysRevApplied.11.064023 (2019).

64. Sitaram, A. et al. Confinement of an alkaline-earth element in a grating magneto-optical trap. Rev. Sci. Instruments 91, 103202, DOI: 10.1063/5.0019551 (2020).

65. Johnson, K. C. Grating Diffraction Calculator GD-Calc ${ }^{\circledR}$, DOI: 10.24433/CO.7479617.v3.

66. Steck, D. A. Rubidium 87 D Line Data. http://steck.us/alkalidata.

67. McGilligan, J. P., Griffin, P. F., Riis, E. \& Arnold, A. S. Diffraction-grating characterization for cold-atom experiments. J. Opt. Soc. Am. B 33, 1271-1277, DOI: 10.1364/JOSAB.33.001271 (2016).

68. Cotter, J. P. et al. Design and fabrication of diffractive atom chips for laser cooling and trapping. Appl. Phys. B 122, DOI: 10.1007/s00340-016-6415-y (2016).

69. Little, B. J. et al. A passively pumped vacuum package sustaining cold atoms for more than 200 days (2021). arXiv: 2101.01051.

70. DeRose, C. T., Trotter, D. C., Zortman, W. A. \& Watts, M. R. High Speed Travelling Wave Carrier Depletion Silicon Mach-Zehnder Modulator. In Opt. Interconnects Conf., 135-136, DOI: 10.1109/OIC.2012.6224486 (SPIE, 2012).

71. Ju, H. et al. Soa-based all-optical switch with subpicosecond full recovery. Opt. Express 13, 942-947, DOI: 10.1364/ OPEX.13.000942 (2005).

72. Zhao, J. et al. Shallow-etched thin-film lithium niobate waveguides for highly-efficient second-harmonic generation. Opt. Express 28, 19669-19682, DOI: 10.1364/OE.395545 (2020).

73. McGilligan, J. P., Griffin, P. F., Riis, E. \& Arnold, A. S. Phase-space properties of magneto-optical traps utilising micro-fabricated gratings. Opt. Express 23, 8948-8959, DOI: 10.1364/OE.23.008948 (2015).

74. Butts, D. L., Kinast, J. M., Timmons, B. P. \& Stoner, R. E. Light pulse atom interferometry at short interrogation times. J. Opt. Soc. Am. B 28, 416-421, DOI: 10.1364/JOSAB.28.000416 (2011).

75. Mielec, N. et al. Atom interferometry with top-hat laser beams. Appl. Phys. Lett. 113, 161108, DOI: 10.1063/1.5051663 (2018).

76. Peters, A. High precision gravity measurements using atom interferometry. Ph.D. thesis, Stanford University (1998). 


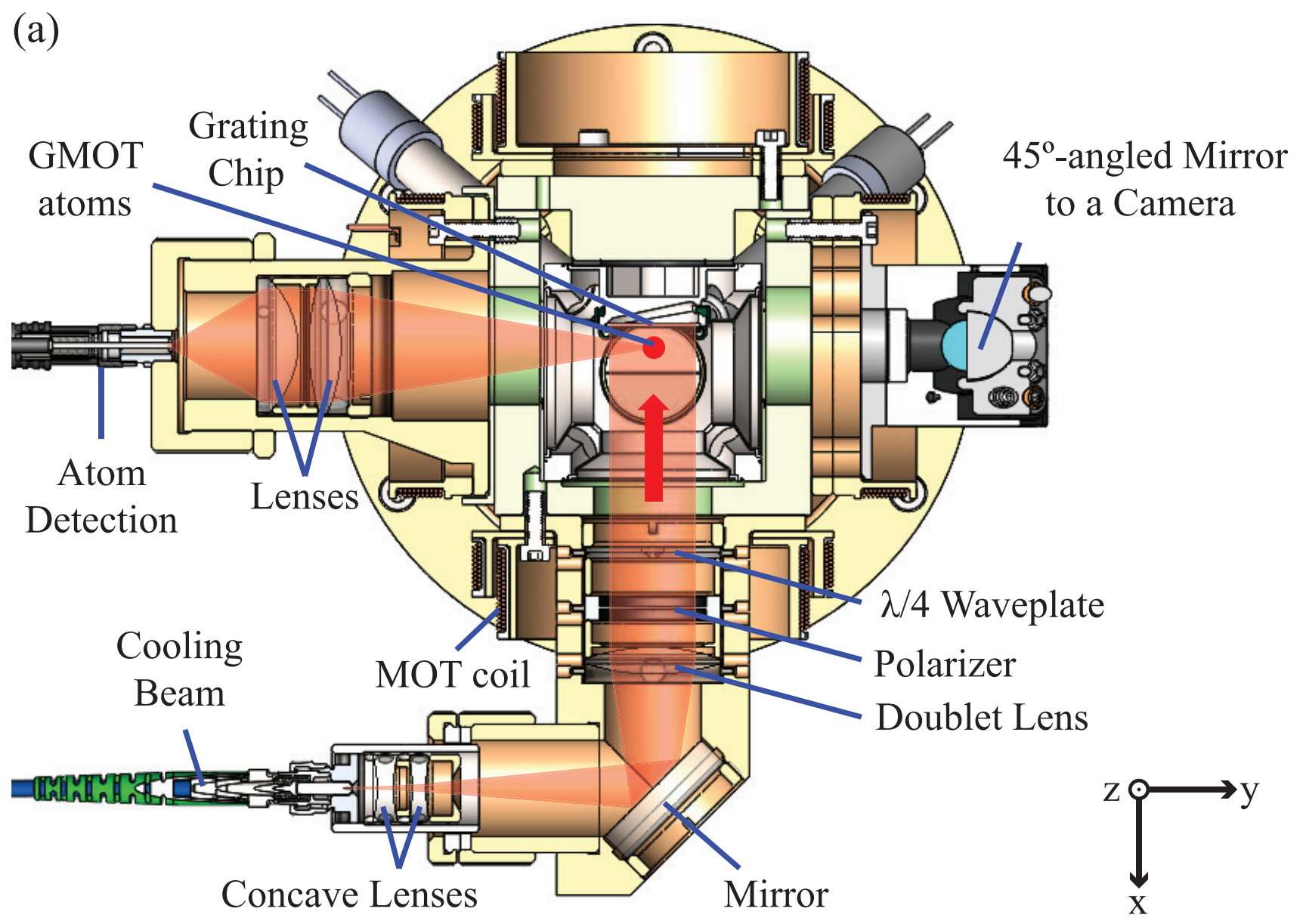

(b)

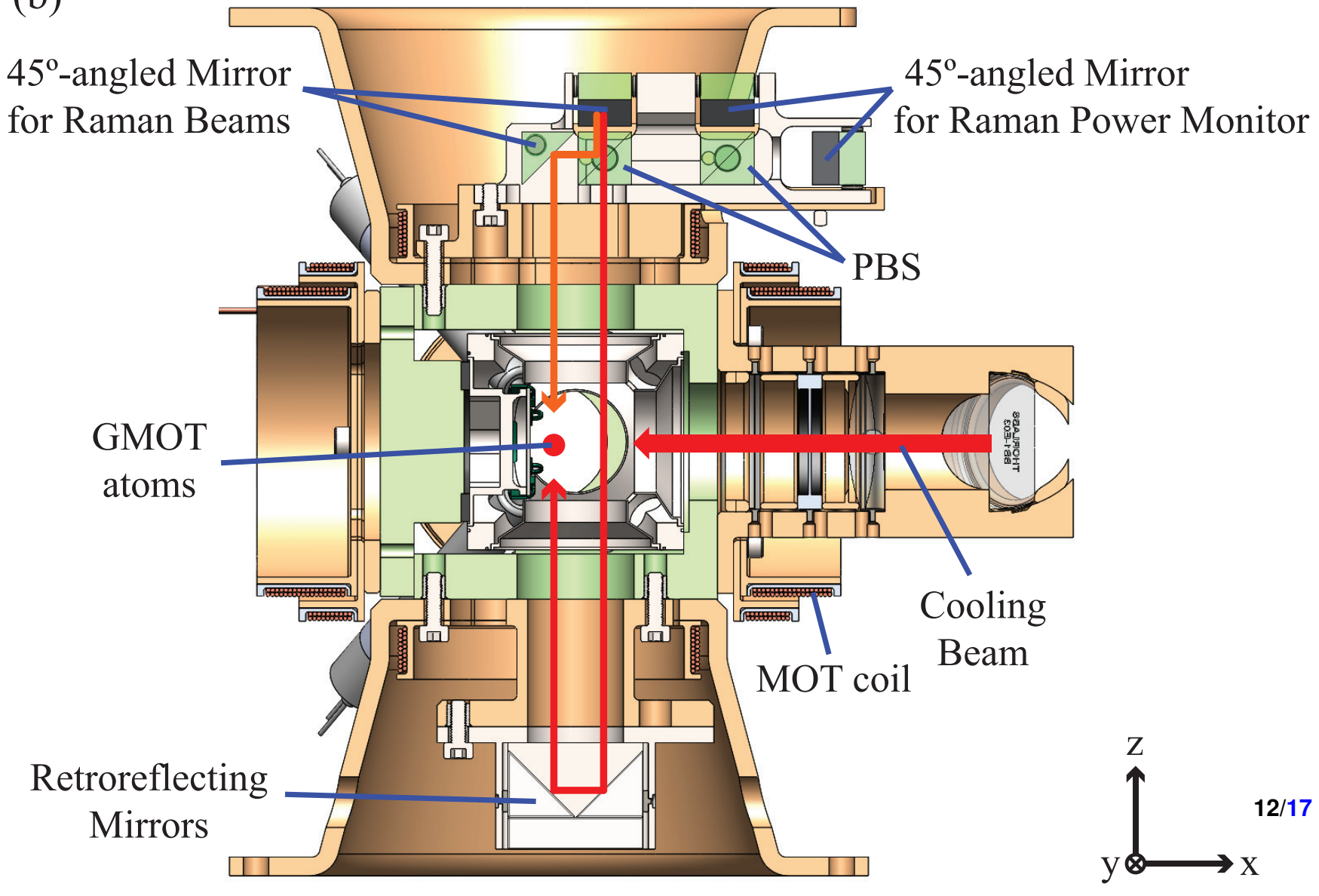


(a)

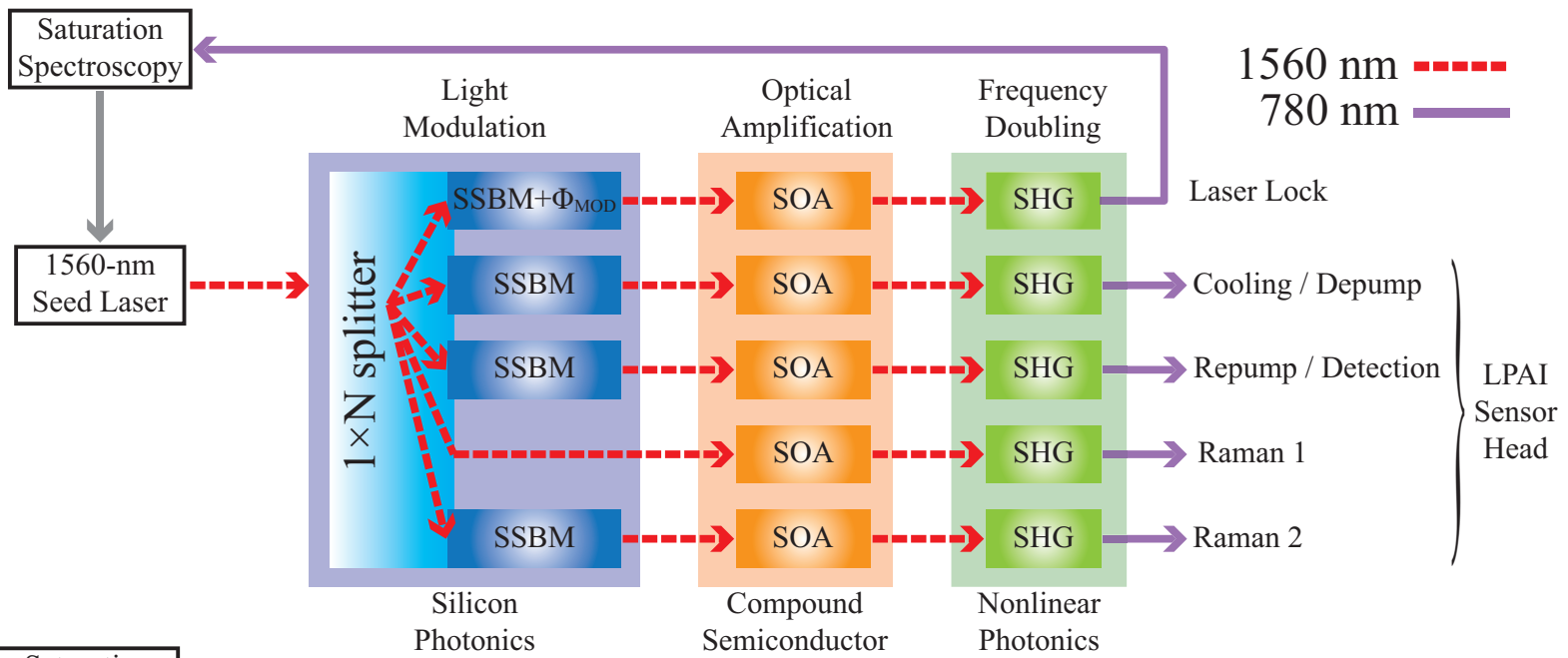

(b)

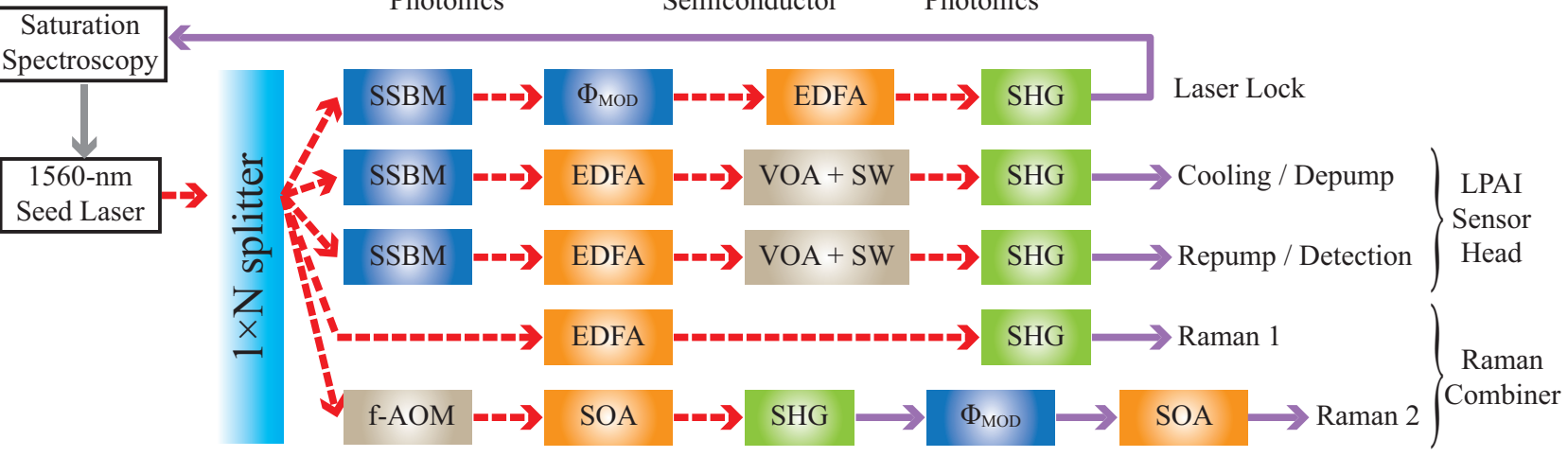

Figure 5. The laser architecture of the LPAI with a single seed laser and time-multiplexed frequency shifting. (a) PIC-based laser system composed of silicon photonics for light modulation, compound semiconductor for optical amplification, and nonlinear photonics for frequency doubling towards a co-packaged laser system and a single hybrid-integrated PIC laser system. SSBM, single sideband modulator; $\Phi_{\text {MOD }}$, phase modulator; SOA, semiconductor optical amplifier; SHG, second harmonic generator. Each channel in silicon photonics include a VOA to control the amplitude of the light, and an SOA can function as an optical switch. (b) PIC-compatible laser system based on discrete components used for the data shown in this paper. f-AOM, fiber acousto optic modulator; EDFA, erbium doped fiber amplifier; VOA, variable optical attenuator; SW, optical switch. For Raman 2, we used a f-AOM at $1560 \mathrm{~nm}$ and a $\Phi_{\mathrm{MOD}}$ at $780 \mathrm{~nm}$, which can be replaced with a SSBM at $1560 \mathrm{~nm}$ similar to the PIC-based laser system. The f-AOM is used for the phase lock by continuously monitoring the beat note between the two Raman beams. Fig. 7 shows the beat note measurement and the switching of the Raman beams. 
(a)

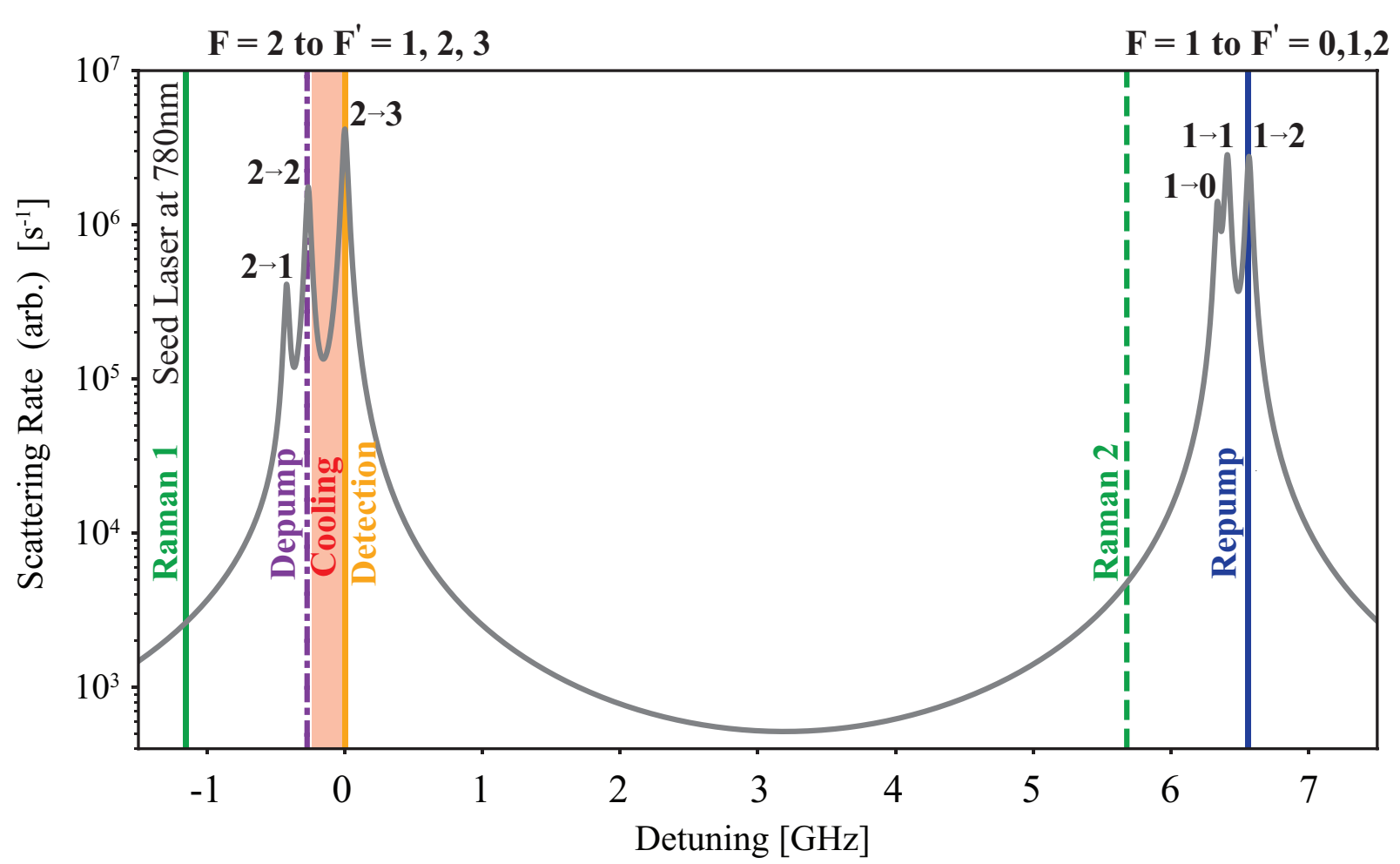

(b)
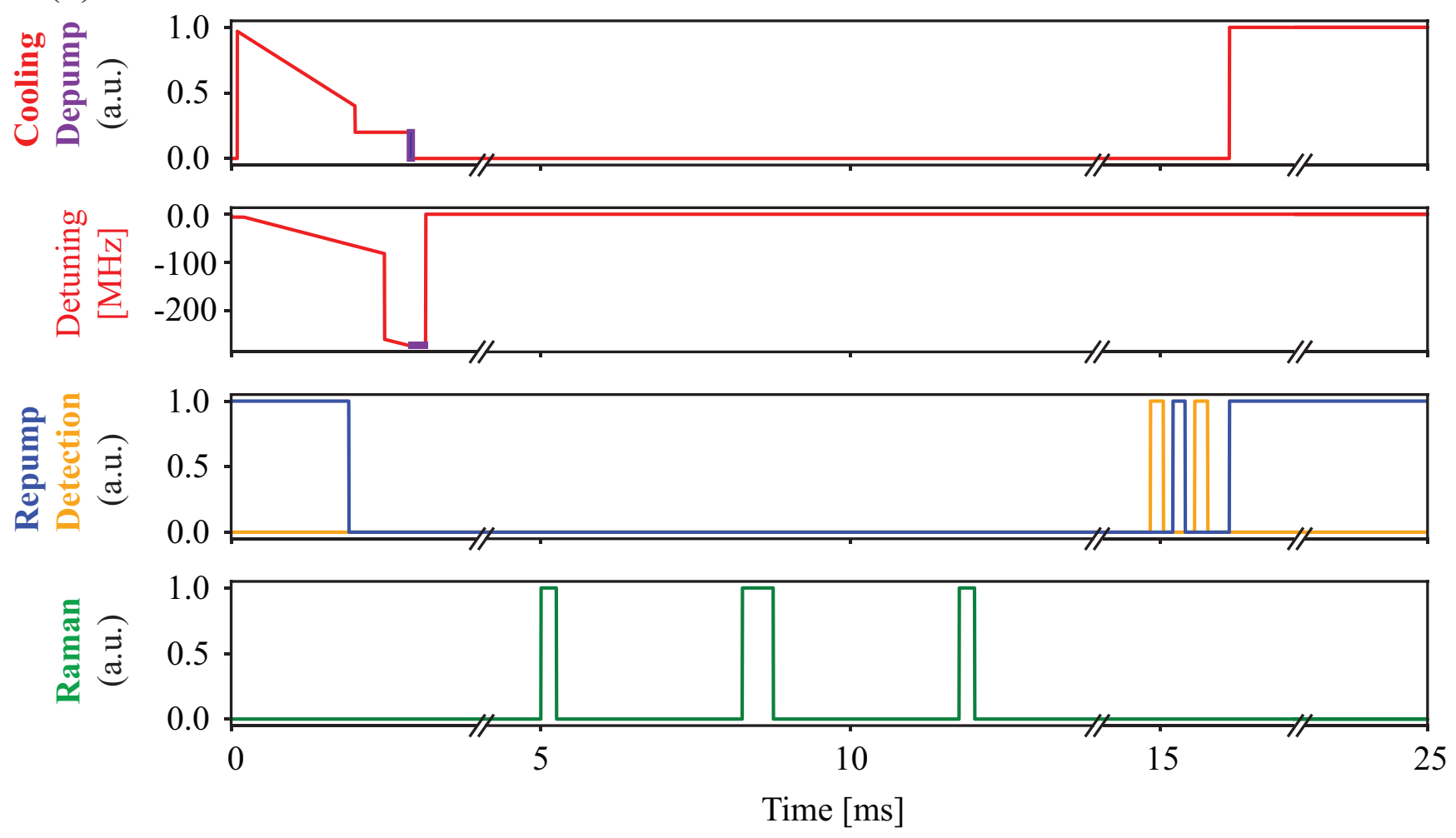

Figure 6. Optical frequency (a) and timing diagram (b) for the PIC-compatible laser system. (a) Optical transitions of ${ }^{87} \mathrm{Rb}$ atoms (D2 transition) ${ }^{66}$. Cooling, Detection, Depump, and Raman 1 beams use $|F=2\rangle$ to $\left|F^{\prime}=1,2,3\right\rangle$ transitions (Left peaks), and Repump and Raman 2 beams use $|F=1\rangle$ to $\left|F^{\prime}=0,1,2\right\rangle$ transitions (Right peaks). The $1560 \mathrm{~nm}$ seed laser is frequency-doubled to $780 \mathrm{~nm}$ and locked to $|F=2\rangle$ to $\left|F^{\prime}=2,3\right\rangle$ crossover transition with $\sim 1.15 \mathrm{GHz}$ red-detuning from the $|F=2\rangle \rightarrow\left|F^{\prime}=3\right\rangle$ resonance, which is also used for Raman 1. (b) Diagram of the timing sequence for LPAI operation highlighting important dynamic changes in the laser channels. The pulse width of Repump/Detection/Raman is not-to-scale. 


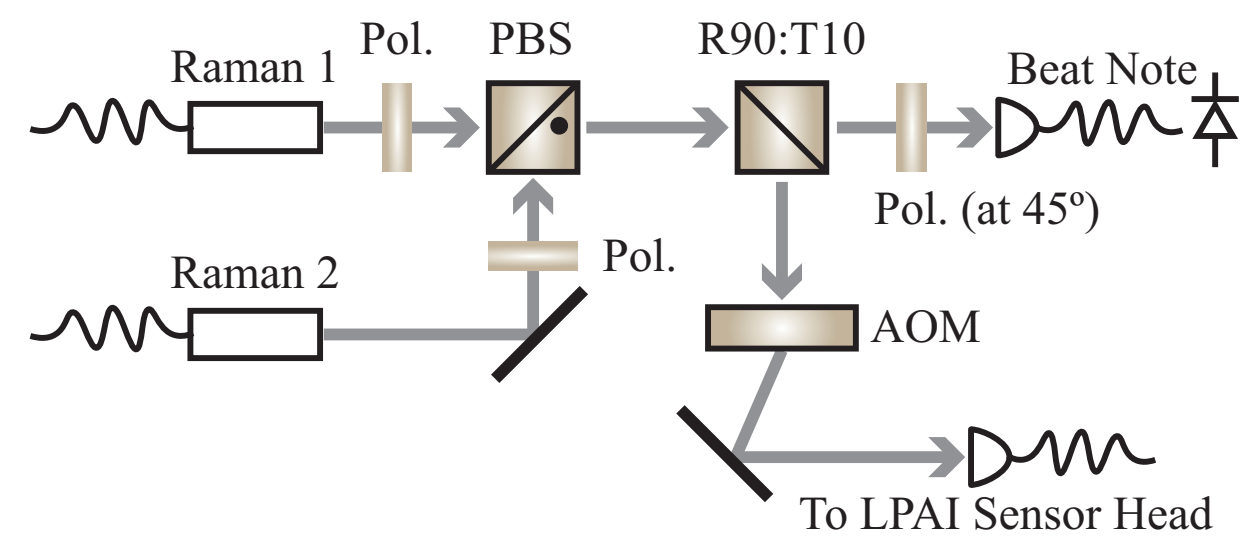

Figure 7. Optical setup for combining the two $780 \mathrm{~nm}$ Raman channels with crossed-linear-polarization and phase locking. Approximately $10 \%$ of the combined power is diverted for phase locking while the remaining power is directed towards an acousto optic modulator for pulsing. The combined Raman beams are coupled into the same polarization maintaining fiber with the polarizations aligned to the slow and fast axes of the fiber. PBS is polarizing beam splitter, R90:T10 is non-polarizing cube beam splitter, Pol. is polarizer, and AOM is acousto optic modulator.
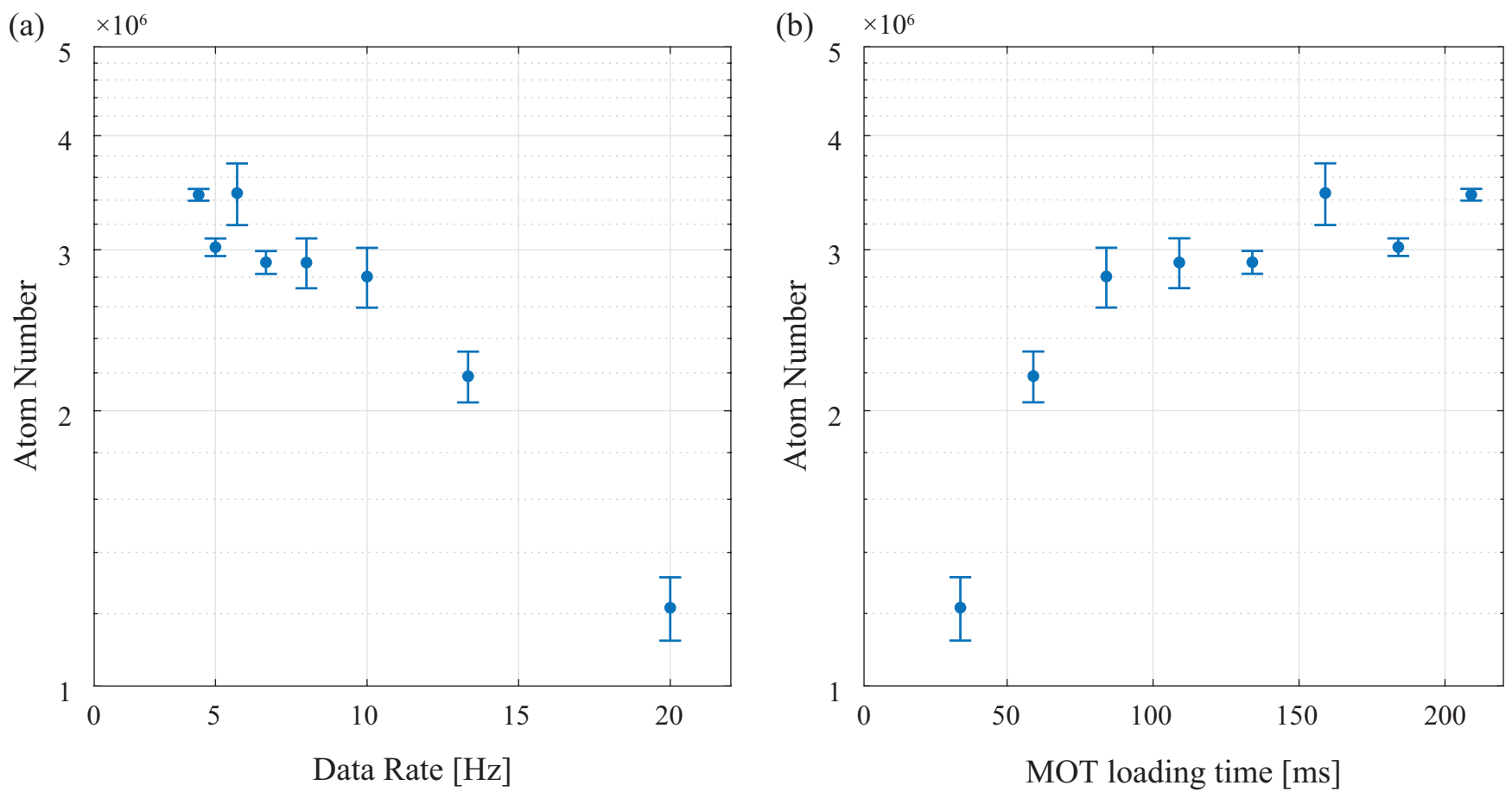

Figure 8. Data rate of GMOT operation including sub-Doppler cooling process. (a) Atom number versus data rate of GMOT operation. (b) Atom number versus MOT loading time. 
(a)

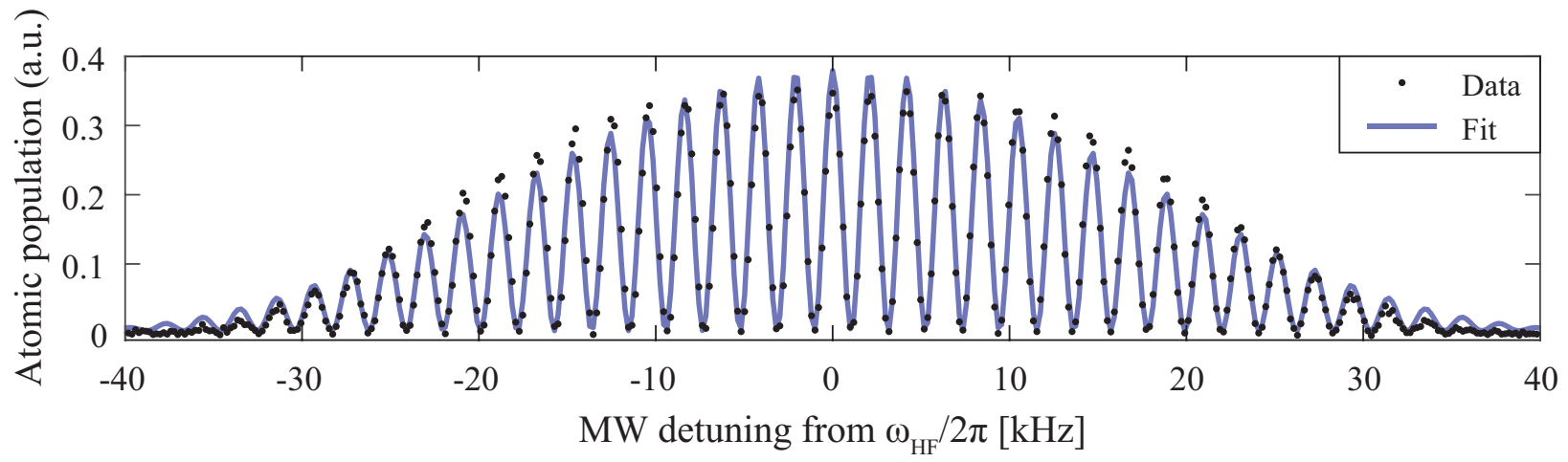

(b)

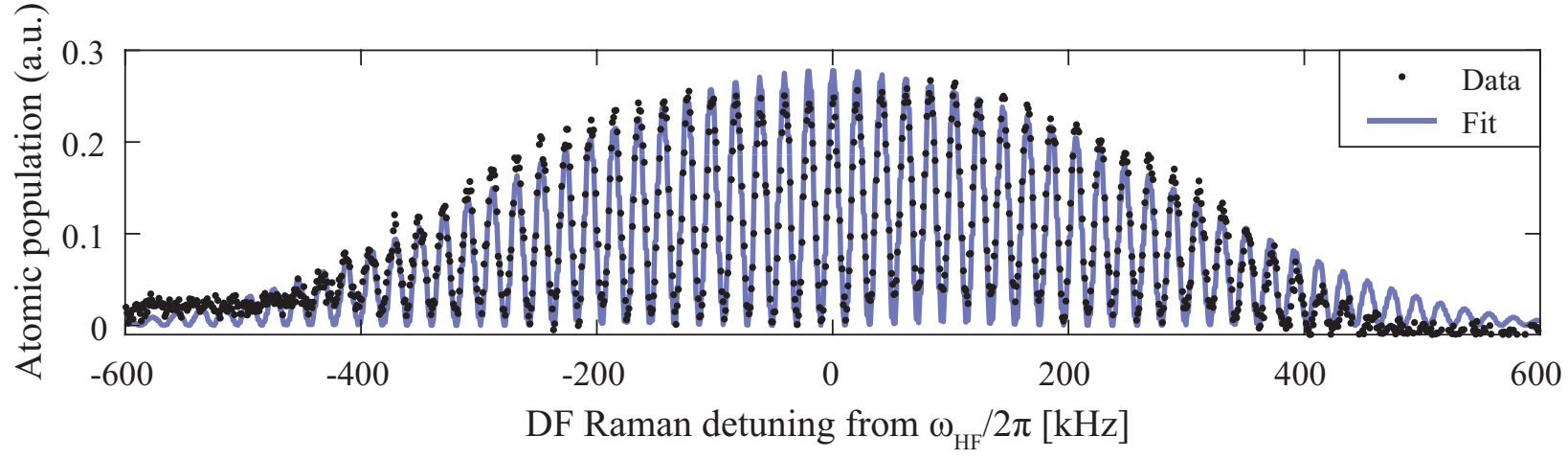

Figure 9. Ramsey interferometry ( $\frac{\pi}{2} \rightarrow T \rightarrow \frac{\pi}{2}$ ) of sub-Doppler cooled GMOT atoms at the compact LPAI sensor head. (a) The atomic fringe of Ramsey interferometry versus microwave detuning from atomic resonance $\left(\omega_{H F} / 2 \pi\right)$. The interrogation time $T$ is $450 \mu$ s, resulting in a fringe spacing of $\delta \omega_{\text {Ramsey }} / 2 \pi \simeq 2.27 \mathrm{kHz}$. The measurement cycle rate is $13.33 \mathrm{~Hz}$. (b) The atomic fringe of Ramsey interferometry versus Doppler-free Raman detuning from atomic resonance $\left(\omega_{H F} / 2 \pi\right)$. The interrogation time $T$ is $48.08 \mu \mathrm{s}$, resulting in a fringe spacing of $\delta \omega_{\text {Ramsey }} / 2 \pi \simeq 19.74 \mathrm{kHz}$. The measurement cycle rate is $10 \mathrm{~Hz}$. 
(a)

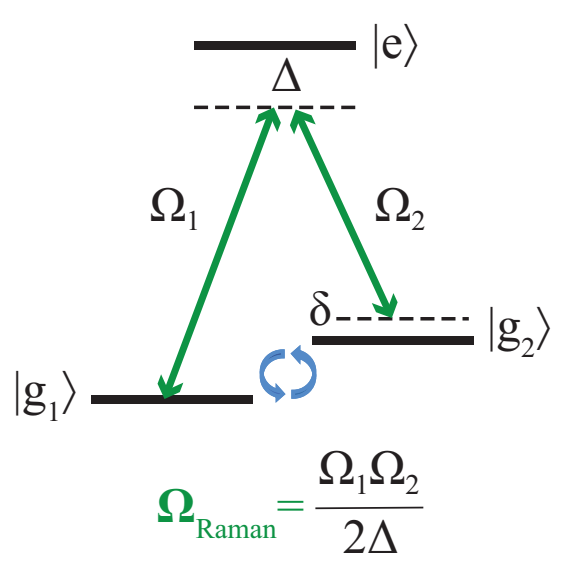

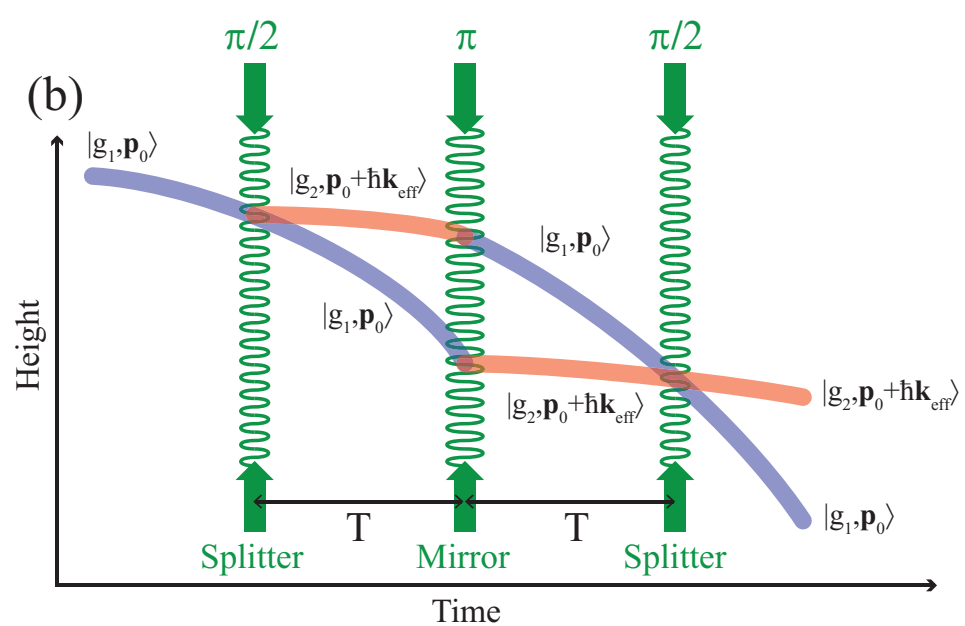

(c)

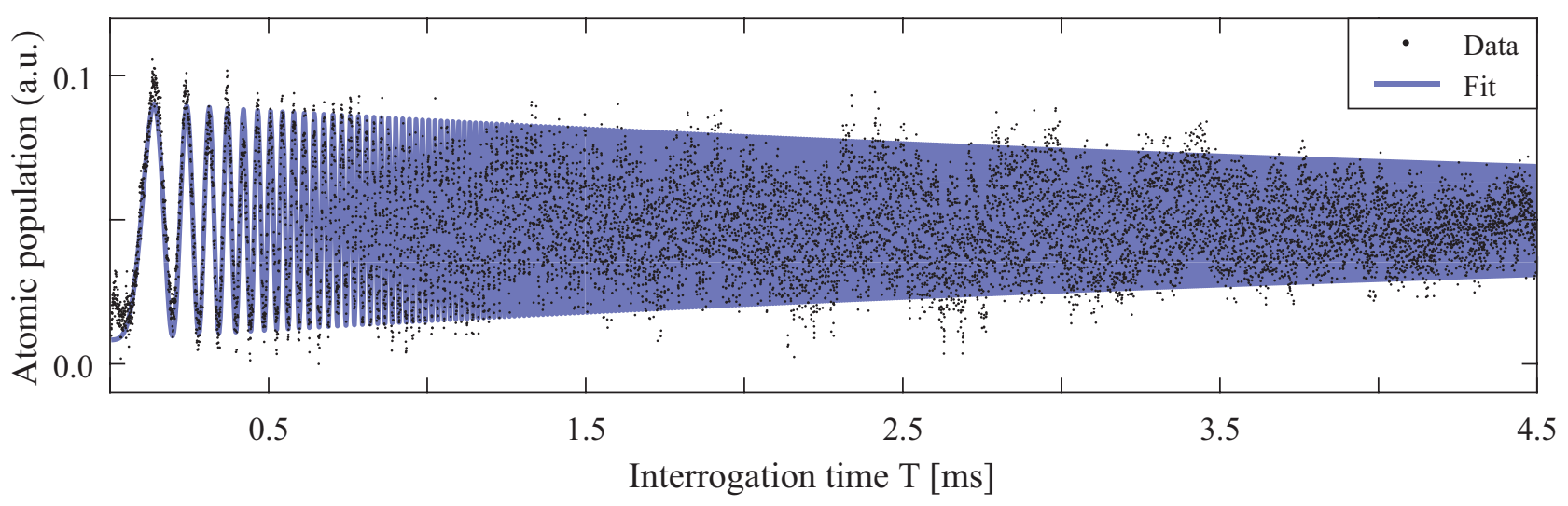

(d)

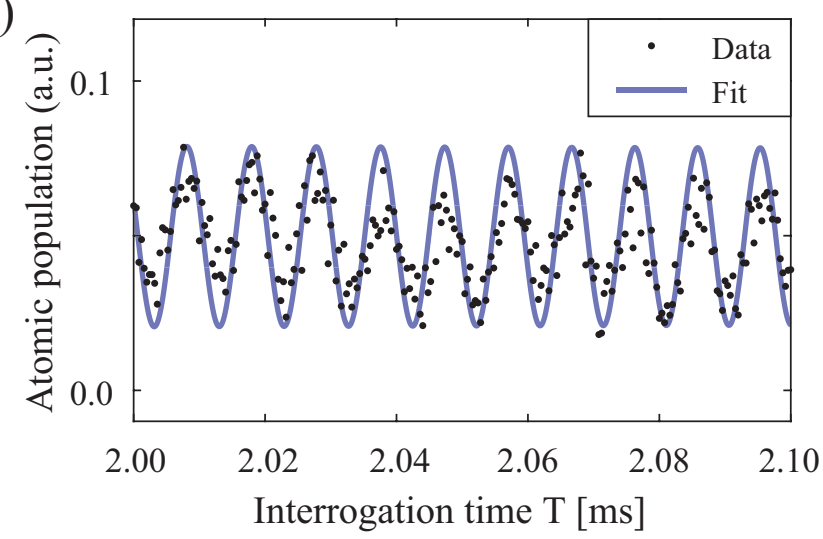

(e)

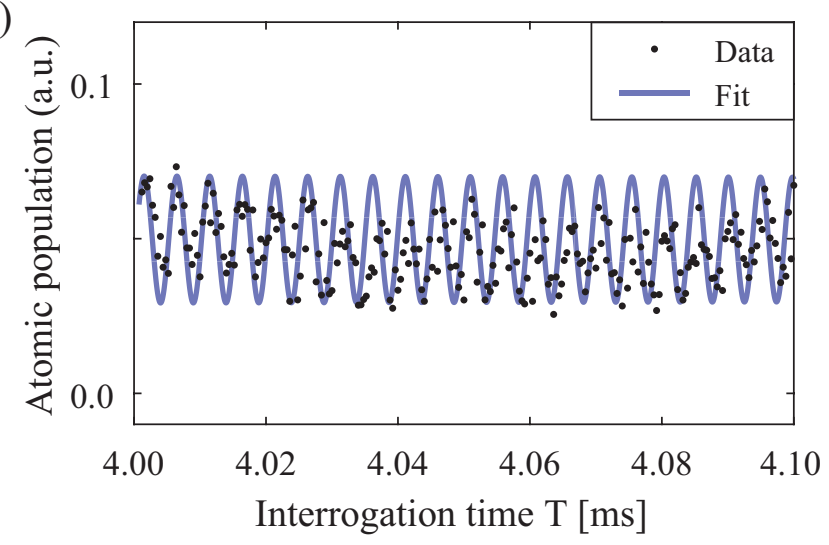

Figure 10. Atom interferometry of sub-Doppler cooled GMOT atoms in the compact LPAI sensor head. (a) Three-level atomic system for stimulated Raman transitions. $\left|g_{1}\right\rangle=\left|F=1, m_{F}=0\right\rangle$, ground state $1 ;\left|g_{2}\right\rangle=\left|F=2, m_{F}=0\right\rangle$ ground state $2 ;|e\rangle$, excited state; $\Delta$, single-photon detuning; $\delta$, two-photon detuning which depends on the Doppler shift; $\Omega_{\text {Raman }}$, effective Rabi frequency of Raman beams; $\Omega_{1}$, single-photon Rabi frequency of $\left|g_{1}\right\rangle \rightarrow|e\rangle$ transition; $\Omega_{2}$, single-photon Rabi frequency of $\left|g_{2}\right\rangle \rightarrow|e\rangle$ transition. (b) Space-time trajectory of atomic wavepackets during LPAI. A three-light-pulse sequence, $\frac{\pi}{2} \rightarrow T \rightarrow \pi \rightarrow T \rightarrow \frac{\pi}{2}$, coherently addresses the ground states of the atoms and provides the state-dependent momentum kicks, $\hbar \mathbf{k}_{e f f} \approx 2 \hbar \mathbf{k}$, where $\mathbf{p}_{0}$ is the initial atomic momentum; $T$, the free-evolution time between Raman pulses; $\mathbf{k}_{e f f}$, the effective wavenumber of stimulated Raman transition; $\mathbf{k}$, the wavenumber $(2 \pi / \lambda)$ of a single Raman beam. (c) Scan of the interrogation time showing the LPAI chirped fringe for $T=0.0 \mathrm{~ms}$ to $4.5 \mathrm{~ms}$. The fringe chirping results from the Doppler shift of the cold atoms relative to the Raman beams as the atoms accelerate due to gravity. Each data point is an average of three points and a slowly-varying offset was removed. The data is fit to a chirped sinusoid ${ }^{76} \cos \left(k_{\text {eff }} g_{k}\left(T^{2}+\tau_{\pi}\left(1+\frac{2}{\pi}\right) T\right)+\phi_{0}\right)$, where $\tau_{\pi}$ is the $\pi$-pulse duration, $g_{k}$ is gravity, and $\phi_{0}$ is an arbitrary phase factor. We measured $g=9.79316(2) \mathrm{m} \mathrm{s}^{-2}$ with the statistical uncertainty of $\Delta g / g=2.0 \times 10^{-6}$ in the condition of no vibration. Plots (d) and (e) provide a detailed view of the chirped fringe of (c) for $T=2.0 \mathrm{~ms}$ to $2.1 \mathrm{~ms}$ and $T=4.0 \mathrm{~ms}$ to $4.1 \mathrm{~ms}$, respectively. The measurement cycle rate is $10 \mathrm{~Hz}$. 